\title{
OPTIMIZATION OF GROWTH PARAMETERS FOR ALGAL REGROWTH POTENTIAL EXPERIMENTS
}

\author{
A Thesis \\ presented to \\ the Faculty of California Polytechnic State University, \\ San Luis Obispo
}

\author{
In Partial Fulfillment \\ of the Requirements for the Degree \\ Master of Science in Civil and Environmental Engineering
}

by

Chadwick Dylan Boggess

June 2014 
(C) 2014

Chadwick Dylan Boggess

ALL RIGHTS RESERVED

Page ii 
TITLE:

AUTHOR:

DATE SUBMITTED:

COMMITTEE CHAIR:

COMMITTEE MEMBER:

COMMITTEE MEMBER:
Optimization of growth parameters

for algal regrwoth potential experiments

Chadwick Dylan Boggess

June, 2014
Dr. Tryg Lundquist, Associate Professor Civil \& Environmental Engineering Department

Dr. Corinne Lehr, Associate Professor Chemistry Department

Dr. Rebekah Oulton, Assistant Professor Civil \& Environmental Engineering Department 


\begin{abstract}
Optimization of growth parameters for algal regrowth potential experiments

Chadwick Dylan Boggess
\end{abstract}

Water cost is one of the key deterrents in making algal biofuels a feasible alternative to traditional petroleum fuels. Research into recycling of algal media was conducted in a laboratory setting at the California Polytechnic State University in San Luis Obispo. A growth assay was created to allow for algal cultures, specifically Ward's Chlorella sp., to proliferate in sealed culture vessels. Bold's Basal Medium (BBM) was selected as the media due to its extensive use with freshwater algal strains. However, this medium can limit algal growth through nutrient limitation and it became necessary to augment the original recipe. $50 \mathrm{mM}$ of sodium bicarbonate was added to the media in order to buffer the purging of $100 \% \mathrm{CO}_{2}$ into the culture vessel. The resultant $\mathrm{pH}$ of the $\mathrm{CO}_{2}$ saturated media was 6.25. The elemental? composition of Chlorella vulgaris was compared with the nutrient content of BBM to estimate possible nutrient limitations which could occur throughout growth (Oh Hama, 1988). From this analysis, the nitrogen and phosphorus concentration in BBM was augmented by a factor of three (BBM3N3P). Further, vitamins, specifically cyanocobalamin, thiamine HCL, and biotin, were incorporated after conducting a comparison growth study with BBM $3 \mathrm{~N} 3 \mathrm{P}$. The average maximum specific growth rates for the normal BBM and for the vitamin-enhanced BBM were $0.689+/$ 0.0818 and $0.887+/-0.1901$ (mean $+/$ - std. deviation) per day, respectively. This difference was proved significant by a t-test with a p-value equal to 0.041 . A standardized methodology for conducting a regrowth potential experiment was outlined by the research. One phase of this methodology included reintroduction of nutrients consumed during the first round of growth. Prior to continuing with this methodology the effect of supplementing trace metals and micronutrients into the culture was investigated. At a p-value of 0.0956, there was no significant difference between BBM $3 \mathrm{~N} 3 \mathrm{P}+$ vitamins and the same media with eight times the trace metal and micronutrient concentration. This allowed for up to seven rounds of regrowth with no inhibition caused from the restoration of these nutrients. A final regrowth was conducted on BBM 3N3P + vitamins $+50 \mathrm{mM}$ bicarbonate. A significant difference existed between both the Round 1 and Round 2 cultures, as well as between the Round 2 cultures and their respective controls. This signified the possible release of allelochemicals by the Chlorella sp.. A preliminary investigation into the possible use of anaerobic digester effluent as a primary source of nutrients for algal cultures was also conducted. The digestate (diluted 50\% v/v with a saline make-up) grew significantly faster than the controls (p-value $<0.001$ ). Further, after the introduction of trace metals and micronutrients the digestate columns outlived the control columns increasing the feasibility of using digestate to supplemental algal cultures.

Keywords: Allelopathy, inhibition, media optimization, digestate 


\section{ACKNOWLEDGMENTS}

I would like to sincerely, and whole heartedly express my gratitude to:

All of the ENVE 405 students for their help throughout the entire project:

Maddie Bouvier, April Oliver, Jaime Middlebrook, Melissa Pasa, Kerry Surjaatmadja, Darren McConnell, Hunter Little,

Trygve Lundquist for accepting me as a graduate student and inspiring me to keep working through the hurdles of algal studies,

Braden Crowe for joining on with the research team, taking a special interest in my project and helping me through the many issues which arose throughout the research,

John Benneman for keeping me in line with his immense algal knowledge and incredible ability to carry out biomass carrying capacity calculations in his head,

Hunter Spence for taking on the next chapter of the research as well as helping me through the entirety of my research,

Alex Hill, Justin Kraetcsh, Eric Nicolai, Nate Olivas, and Mike Chang for being an epic group of graduate students,

Department of Energy for providing food, tuition, and housing for me for the last year and a half,

My parents for supporting my love of mathematics and sciences from a very young age, And finally for all of my friends for helping to keep my sanity, I love all of you! 


\section{TABLE OF CONTENTS}

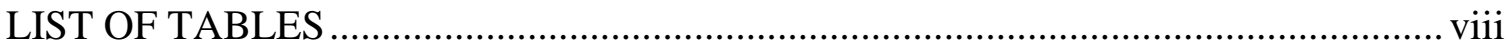

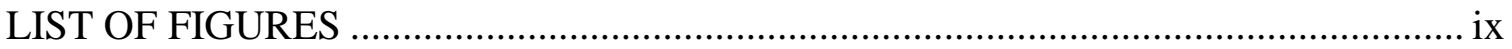

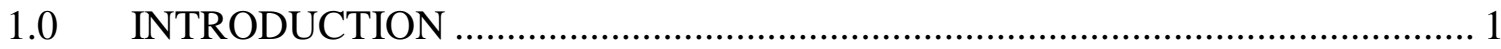

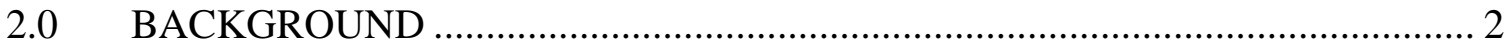

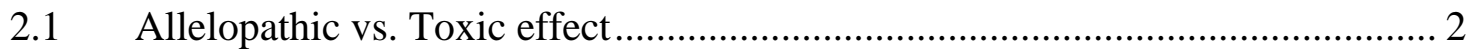

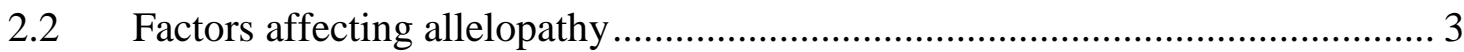

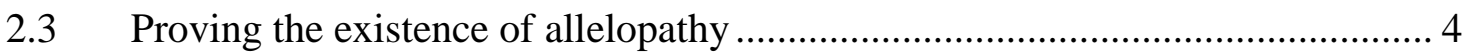

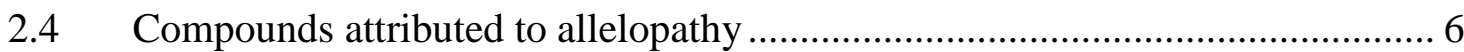

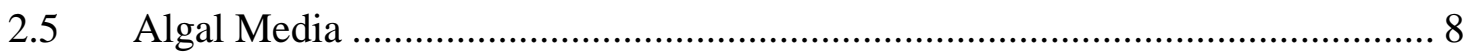

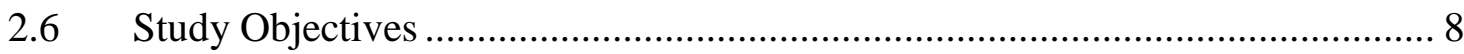

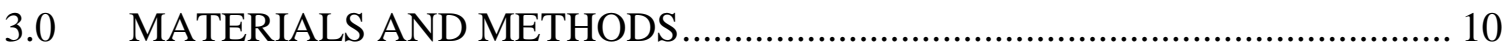

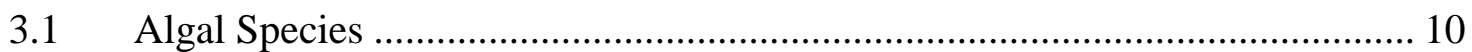

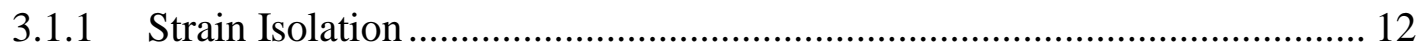

3.2 Media Preparation ........................................................................... 12

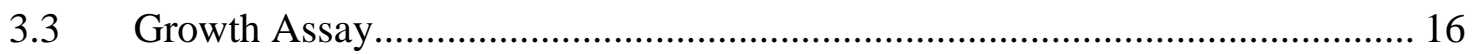

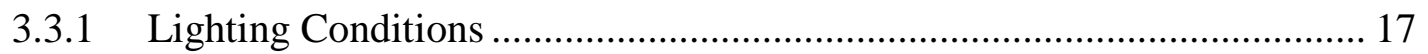

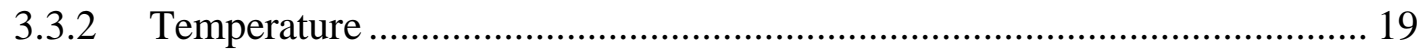

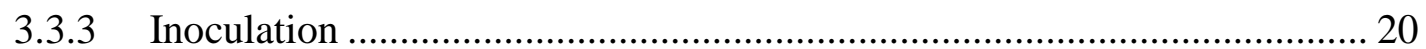

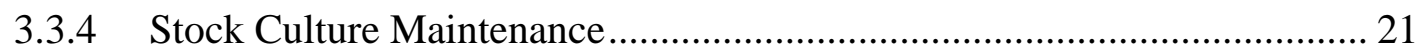

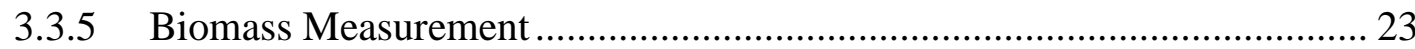

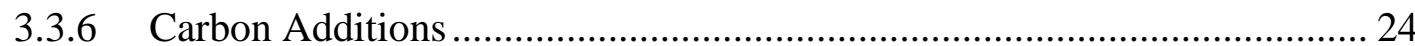

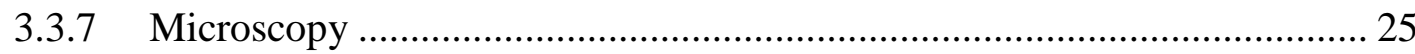

3.4 Recycled Media Experimental Design...................................................... 25

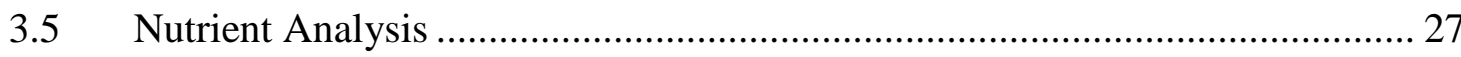

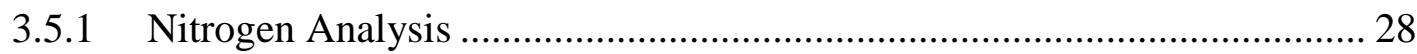

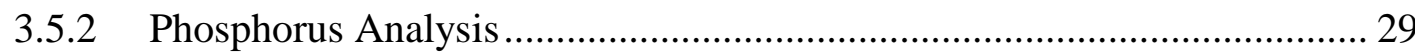

3.6 Nutrient Replenishment ........................................................................... 30

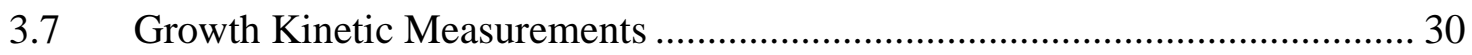

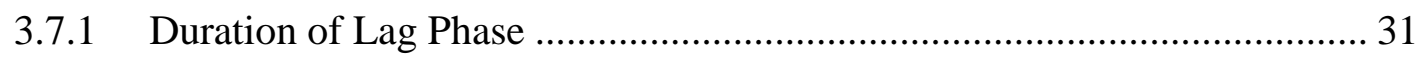

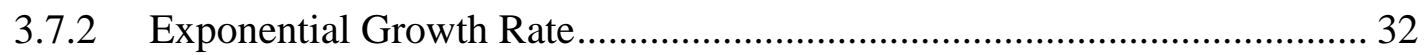

3.7.3 Light Limited Growth Rate................................................................ 32

3.8 Digestate Effluent Regrowth Experiments ..................................................... 33

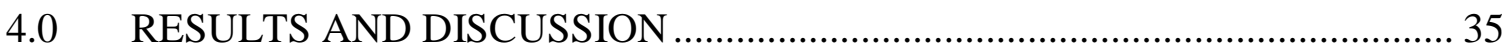




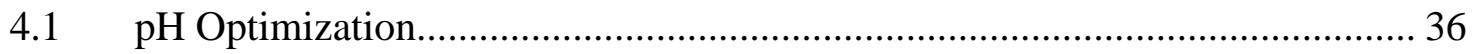

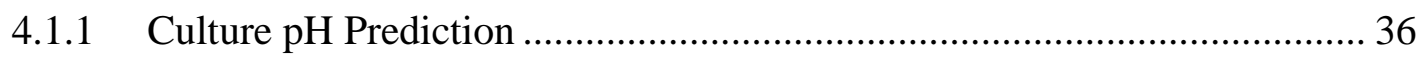

4.1.2 Carbon Dioxide Saturation Experiment.................................................. 38

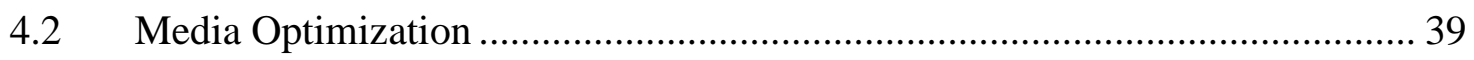

4.2.1 Media Carrying Capacity ....................................................................... 39

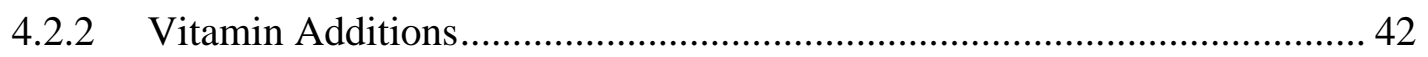

4.3 Trace Metal and Micronutrient Spike ……….............................................. 44

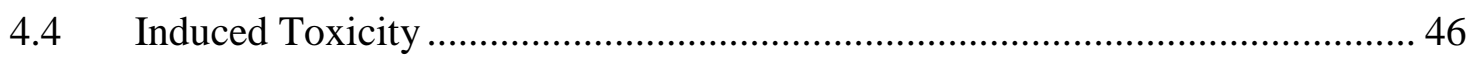

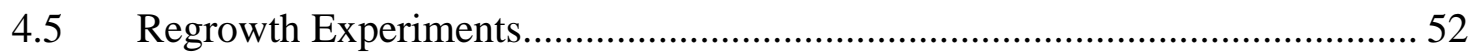

4.5.1 Regrowth on Bold's Basal Medium (3N3P) with Vitamins and

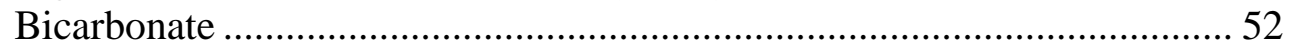

4.5.2 Regrowth on Anaerobically Digested Lysed Algal Biomass ..................... 58

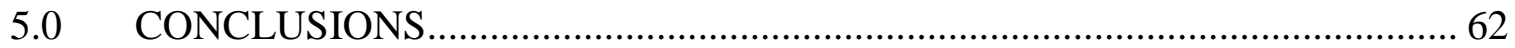

5.1 Bold's Basal Medium (3N3P with Vitamins and Bicarbonate) Regrowth

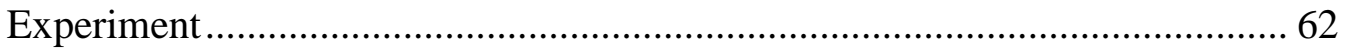

5.2 Anaerobically Digested Algal Biomass Growth Potential Experiment ............ 62

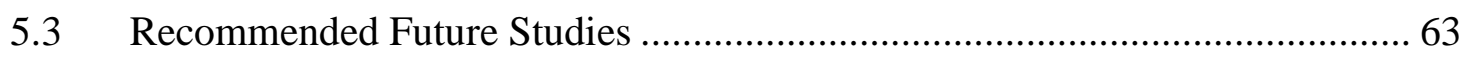

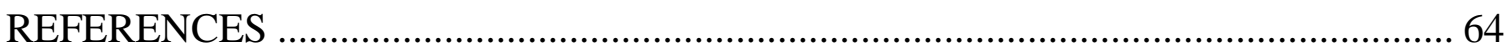

APPENDICES

Appendix A : Summary of Spreadsheet Titles of Experimental Data........................... 66 


\section{LIST OF TABLES}

Table 1: Nutrient constituents of Bold's Basal Medium for both 1N1P and 3N3P. $1 \mathrm{~N} 1 \mathrm{P}$ denotes one times the nitrogen and phosphorus content traditionally found in BBM media whereas 3N3P denotes three times the normal molar concentrations. Each constituent was stored in a separate, capped Pyrex bottle. Each constituent was added in the order listed when preparing the media. (NCMA Bold's Basal Medium) The volumes of each stock solution which were added to create one liter of Bold's Basal Medium were also included.

Table 2: Vitamin concentrations of the modified Bold's Basal Medium used after January 2013. Each vitamin stock solution was prepared with HEPES buffer and adjusted to $\mathrm{pH}$ 7.8. Vitamins were stored in the freezer to preserve the vitamins and protect from heat and light degradation. 15

Table 3: Data collected from the trace metal spike experiment. C1-4 refer to the control cultures, while T1-4 refer to the experimental cultures with $8 x$ micronutrients. Experiment 1 refers to the plotted data from 0-50 hours. At that time, the cultures were discarded, the bottles refilled with fresh media, and reinoculated, marking the beginning of Experiment 2 which extended from hours 50-72.

Table 4: Data gather from $\mathrm{Cu}$ induced inhibition of the ISO strain. The $\mathrm{p}$ value obtained from the student $t$-test confirmed that a significant difference exists between the control and test cultures. C1-3 refer to the control cultures while T1-3 refer to the test cultures. 


\section{LIST OF FIGURES}

Figure 1: Effect of light, $\mathrm{pH}$, and nutrients on the effectiveness of allelopathy

(Graneli, 2006).

Figure 2: Summary of identified allelopathic compounds produced by freshwater algae. Effects and mode of action was included for select compounds (Leflaive, 2007).

Figure 3: A photomicrograph showing Ward's Chlorella sp. The micrometer burnt into the image measures 50 micrometers. [1000x total magnification, brightfield]

Figure 4: Photomicrograph of the isolated algal strain taken from wastewater treatment ponds in San Luis Obispo, CA. [400x total magnification, brightfield]

Figure 5: Graphic of the algae growth vessels. A pyrex glass bottle was sealed with a silcon septa and an aluminum crimp cap. Experimental culture volume was $200 \mathrm{~mL}$ with the remaining $80 \mathrm{~mL}$ composed of pure carbon dioxide.

Figure 6: Light map generated for the incubator lighting array using the Li-COR sensing instrument.

Figure 7: As temperature increases from $10-25^{\circ} \mathrm{C}$ the growth rate of Chlorella vulgaris also increases. $25^{\circ} \mathrm{C}$ was chosen as the target temperature for this research due to the high growth rates and mimicry of the ambient environment of San Luis Obispo, CA.

Figure 8: The solid line depicts the absorbance spectrum of Chlorella vulgaris grown with water. The absorbance declines rapidly above a wavelength of $730 \mathrm{~nm}$.

Figure 9: Carbon purging system for algae growth vessels. Culture was agitated using a stir plate while $100 \% \mathrm{CO} 2$ was pumped into the headspace through the sterile syringe filter. An oxygen and carbon dioxide mixture exited the growth vessel through the second sterile needle.

Figure 10: This figure shows a flowchart which describes the process of growing, harvesting, analyzing, restoring, and reinoculating for regrowth experiments.

Figure 11: Graphic depicting the classic growth curve for algae. For this research the main areas of focus included the lag, exponential, and stationary phase. This graphic does not depict the light limited growth region which occurs as the transition between the exponential and stationary phases. Adopted from Bacterial Growth and Regulation, 2007.

Figure 12: Flowchart describing the various experiments conducted to minimize the influence of external factors on growth. The orange boxes indicate calculations while the green boxes depict experiments that were conducted. 
Figure 13: Predicted $\mathrm{pH}$ after $\mathrm{CO}_{2}$ saturation based on bicarbonate concentration

Figure 14: Graphic showing the resultant $\mathrm{pH}$ of the culture media after the introduction of various concentrations of sodium bicarbonate and saturation with gaseous carbon dioxide

Figure 15: Graphic of the generated biomass carrying capacity spreadsheet used in analysis of nutrient limitation as well as nutrient restoration between rounds of regrowth experiments.

Figure 16: Plotted growth data from comparison study between normal and vitamin-enhanced Bold's Basal Medium. The top figure shows a normal OD vs. time, while the bottom figure shows the optical density on a logarithmic scale. $\mathrm{N}$ denotes the control cultures which contained the original BBM (1N1P) recipe while $\mathrm{V}$ denotes the cultures which had vitamins added.

Figure 17: Plotted growth data from the trace metal spike experiment. The graphic on the left plots OD vs. Time while the graphic on the left shows a logarithmic plot of OD vs. Time. The experiment was restarted at a time equal to 50 hours.

Figure 18: Induced inhibition test using $469 \mathrm{ppb}-\mathrm{Cu}$ on an algal strain isolated on BBM media with $1.7 \%$ agar from the California Polytechnic State University's wastewater treatment ponds. C1-3 refer to the control cultures while T1-3 refer to the test cultures.

Figure 19: The growth curves for the third induced inhibition experiment. The controls are red while the experimental cultures are blue.

Figure 20: Level of $\mathrm{Cu}$ addition and the observed maximum specific growth rate (per day). The highlighted growth rate was determined to be an outlier and omitted from the analysis of the experiment (resides farther than 1.5 times the interquartile range).

Figure 21: The exponential growth rates for each test culture were normalized to the average exponential growth rate of the control cultures. The resulting graphic shows a decreasing normalized growth rate from 5 to $10 \mathrm{ppm}$ $\mathrm{Cu}$.

Figure 22: Round 3 of growth during a preliminary regrowth experiment. From left to right: $\mathrm{x} 3$ controls, $\mathrm{x} 2$ experimental cultures with only nitrate and phosphate restored, $\mathrm{x} 3$ experimental cultures with nitrate, phosphate, and trace nutrients restored.

Figure 23: Round 1 growth of experimental cultures A1-10. Cultures were inoculated on March 31 and harvested on April 6, 2014.

Figure 24: Round 2 growth data of cultures A1-10. They were separated into A1-5 and A6-10 due to the cultures residing on different stir plates. Each group of five experimental bottles and had an equal number of controls on the respective stir plate.

Figure 25: Various stages of harvesting of Round 1 algae cultures grown in BBM (3N3P + Vitamins + Bicarbonate) (A1-10). From left to right: unfiltered, centrifuge supernatant, 0.45 micrometer filtered. 
Figure 26: Growth rates determined for the experimental cultures for both round 1 and 2 of growth. The growth rates were normalized to the average growth rate for controls to allow for unbiased comparison. Round 2 growth rates were outlined with a black border for distinction.

Figure 27: Various stages of anaerobically digested algal biomass. From left to right: 0.22 micrometer filtered, 0.45 micrometer filtered, 1.1 micrometer filtered.

Figure 28: Photo showing light rack after inoculation of digestate regrowth experiment. From left to right: $\mathrm{x} 3$ Nannochloropsis maintenance cultures, $\mathrm{x} 2$ HTL, $\mathrm{x} 5$ controls, $\mathrm{x} 5$ digestate columns (50\% v/v dilution), $\mathrm{x} 2$

Nannochloropsis maintenance cultures.

Figure 29: Columns on day 4. Note the discoloration associated with the digestate columns. Photo taken on April 4, 2014.

Figure 30: Growth data collected during the digestate growth potential experiment. Blue denotes control cultures while red denotes experimental cultures with $50 \%(\mathrm{v} / \mathrm{v})$ digestate.

Figure 31: Photograph of the digestate columns on April 17, 2014. The columns gained the dark, green color after the addition of trace metals and micronutrients. 


\subsection{INTRODUCTION}

Current trends in population growth combined with economic development are demanding expansion of our current energy economy. However, due to the environmental liabilities of fossil fuel combustion, biofuels are promoted as a potentially more environmentally-sustainable alternative. Distinct from the many terrestrial biofuel feedstock crops such as corn and waste feedstocks such as municipal solid waste, microalgae are seen to have great potential as a fast-growing feedstock (Moody J., 2014). Unfortunately, like other crops, microalgae cultivation consumes water via evaporation and nutrients via volatilization and other pathways, and these inputs degrade the sustainability of algae biofuel (NRC 2012). Recycling algae growth media, following algae harvesting, is one way to likely improve the sustainability algae biofuels, and the present thesis focuses on media recycling A key deterrent in the use of algae in production of biofuels stems from the requirement of water for the algal media. This is mainly due to the high associated cost as well as the current widespread scarcity of freshwater. This research aims to

generate an optimized methodology for conducting regrowth experiments on algae to determine if recycling of algal media through successive rounds of growth will have an effect on the productivity of the algae system. This research also aims to determine, on a preliminary scale, if anaerobic digester effluent can be used as a nutrient slurry for algal media. 


\subsection{BACKGROUND}

A summary of pertinent research surround allelopathy, algal media, and batch growth kinetics was included and discussed in the following section.

\subsection{Allelopathic vs. Toxic effect}

Commonly, allelopathic chemicals and toxins are grouped together under the same category of compounds. However, allelopathic compounds are noted for their effect on direct predators or competitors while toxins are noted for their effect on a wide array of organisms (Leflaive, 2007). Using this as a basis for differentiating between toxins and allelopathic compounds, allelopathy can be defined as an inhibitory effect caused by generated secondary metabolites that act against direct competitors or predators.

Most allelochemicals have been characterized by their activity spectra, molecular weights, and sometimes their gross chemical characteristics but rarely by their chemical structures (Legrand, 2003). Determining the mode of action is difficult with an incomplete image of the compound. However, several generalized modes of action through which allelopathy can occur have been outlined. These include inhibition of photosynthesis, nucleic acid synthesis, enzyme production, reactive oxygen species (ROS) generation, or paralysis of the complete cell (Leflaive, 2007).

Inhibition of photosynthesis is accomplished through the infiltration of the allelopathic compounds to the thylakoid membrane. Properties of these compounds include low molecular weights, insolubility in water, and solubility in non-polar? organic solvents. All of these properties aid the compounds in reaching the membrane where they have been noted to inhibit photosystem II-mediated photosynthesis (Gleason \& Baxa, 1986; von Elert \& Juttner, 1996, 1997) Nucleic acid synthesis is interrupted through targeting 
of RNA polymerase. This has been a noted effect against bacteria, fungi, and green algae (Doan N.T., 2000). Through evolution, aquatic organisms have adapted to producing extracellular enzymes to aid in metabolism. By targeting the production of these enzymes, the nutrition entering the cells is severely diminished inhibiting growth rate (Juttner F, 2000). Motility is a huge advantage for selective species of microorganisms. Cyanobacterium have been observed to induce paralysis, and therefore increase the rate of settling of rival green algae (Kearns K.D., 2000). The final mode of action is through the creation of reactive oxygen species. The production of these species induces intracellular oxidative stress, which can affect enzyme function, cause cytotoxicity, or produce superoxide radical ions (O2-) (Vardi A., 1999).

\subsection{Factors affecting allelopathy}

The production of allelopathic compounds is related to biotic and abiotic factors. The density of both the producing and targeted species is important in order to create a substantial amount of the chemical as well as to have a large enough population to note an allelopathic occurrence.

Similarly, the physical size and shape of the target organism can affect the allelopathic response (Graneli, 2006). Larger cells may increase the contact time between the target organism and the allelopathic compound, or may increase the number of binding sites

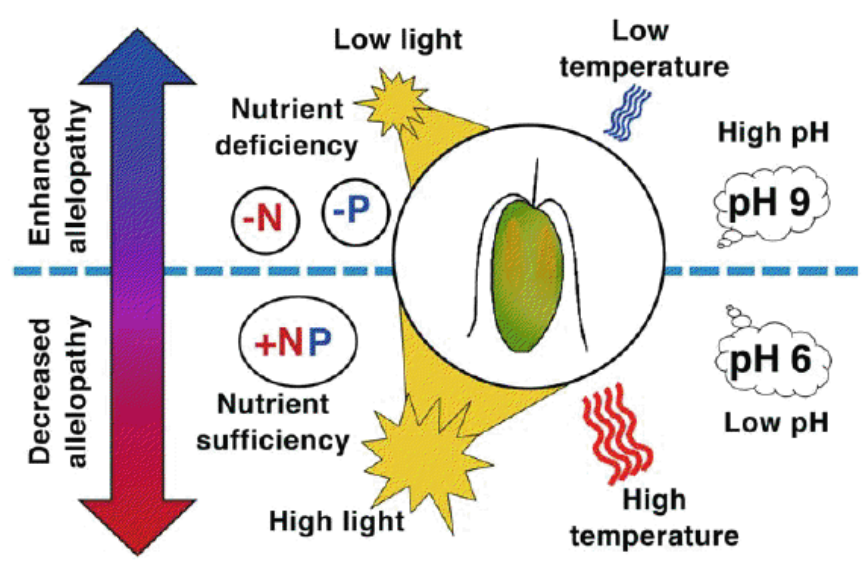

Figure 1: Effect of light, $\mathrm{pH}$, and nutrients on the effectiveness of allelopathy (Graneli, 2006). 
for the compound. Both of these effects would increase the effectiveness of the allelopathic compound.

Abiotic factors include those that are dictated by the physical and chemical properties of the environment, specifically the media with respect to microorganisms. A limited amount of research has been conducted regarding the specific effect of abiotic factors such as light, media composition, and $\mathrm{pH}$ on allelopathy (Graneli, 2006). Light is essential to allow the proliferation of phototrophs as it allows the conversion of carbon into sugars and fats. The nutrient content of the media dictates the maximum biomass carrying capacity while the $\mathrm{pH}$ controls the equilibrium between various forms of ions residing in the media. Controlling all of these factors in an outdoor, ambient environment is nearly impossible. Therefore, much of the research surrounding allelopathy is conducted in controlled laboratory settings.

\subsection{Proving the existence of allelopathy}

Along with production of primary compounds such as lipids or sugars, photoautotrophic microorganisms are known to produce many different secondary metabolites (Leflaive, 2007). Due to the immense number of these compounds, only a small number have been identified and their effects categorized. Nearly all of the identified allelopathic compounds were identified through research in a laboratory setting. This is a direct effect of the difficulty of controlling the ambient environment enough to allow for the sensitivity required to observe, identify, and confirm allelopathy. In 1985, Willis outlined 6 criteria to effectively demonstrate allelopathy occurring in higher plants: “(1) a consistent pattern of inhibition of the target species must be seen; (2) the inhibitor species must produce a toxic chemical; (3) a mechanism for toxic chemical 
release into the environment must be present; (4) there must be a system for transporting or accumulating the toxic chemical within the environment; (5) the target species must have some means of taking up the toxic chemical; and (6) the pattern of inhibition must not be explainable by physical factors or other biotic factors, especially competition and grazing (Legrand, 2003).” A literature review by Legrand revealed no research effort has been able to prove all six of the criteria. Most likely this is attributed to the large-scale dilution that occurs when compounds are secreted into the ambient environment. Because of this dilution, compounds are not present in concentrations adequate to produce observable inhibitory effects.

Experiments were conducted which focused on mixed growth experiments. These experiments combined a toxic and non-toxic species on a petri dish. As both organisms proliferate, the compounds produced by the toxic organism inhibit or kill the non-toxic organism, resulting in a dead zone. This style of experimentation is common during the design and testing phases of antibiotic creation. The main issue with studying allelopathic interaction is ensuring the observed effect is truly allelopathic in nature. It is impossible to eliminate allelopathic interactions in competition experiments; however, it is possible to eliminate competition from allelopathic interaction experiments (Legrand et al. 2003). This can be accomplished by ensuring there is no limitation on the abiotic growth factors such as light, nutrients, and $\mathrm{pH}$.

Several organizations have outlined methods for detecting toxicity. These methods are typically applied during testing of the toxicity of a compound on a particular organism. OECD, APHA, and the USEPA have all outlined a detailed methodology. Each methodology contains a recommended media recipe, required growth rates, 
recommended transfer frequency, and methods for measuring biomass. Each

methodology refers to testing a specific effect of a compound; however, since the exact substance causing the allelopathy was unknown it was necessary to adapt the methods. Information from all three methods was combined for this research in order to ensure validity and congruency with testing ethics.

\subsection{Compounds attributed to allelopathy}

The various research efforts surrounding allelopathy have turned up several compound's which have been confirmed to cause inhibition to some degree. Compounds produced by freshwater algae which have been observed to inhibit the growth of either bacteria, fungi, or other algae species are listed in Figure 2. Most commonly the effect of the compounds is inhibition of cell division. However, some species produce compounds which cause death in small vertebrates. Other research has concluded that linoleic and linolenic acids as well as their degradation products cause inhibition in green algae. These acids are described as essential fatty acids as humans are not able to synthesize it from various food components (Ikawa, 1997). Other structures noted in allelocompounds include alkaloids, cyclic peptides, terpenes, and volatile organic compounds (Leflaive, 2007). 


\begin{tabular}{|c|c|c|c|c|c|}
\hline Compound & Emitter & Target & Effect & Mode of action & References \\
\hline \multicolumn{6}{|l|}{ Allelopathic compounds } \\
\hline Calothrixine A & Calothrix sp. & Bacteria & Growth inhibition & $\begin{array}{l}\text { Inhibition of RNA synthesis } \\
\text { and DNA replication }\end{array}$ & Doan et al. (2000) \\
\hline \multirow[t]{2}{*}{ Cyanobacterin } & \multirow[t]{2}{*}{ Scytonema hofmanni } & $\begin{array}{l}\text { Aquatic and } \\
\text { terrestrial plants }\end{array}$ & Growth inhibition & PSII inhibition & $\begin{array}{l}\text { Gleason et al. } \\
\text { (1986) }\end{array}$ \\
\hline & & Micro-algae & Growth inhibition & PSI inhibition & Gleason \& Paulson (1984) \\
\hline $\begin{array}{l}2 E, 4 Z \text {-decadienal and } \\
\text { other polyunsaturated } \\
\text { aldehydes }\end{array}$ & $\begin{array}{l}\text { Thalassiosira rotula and } \\
\text { other diatoms }\end{array}$ & Invertebrates & $\begin{array}{l}\text { Decrease in fecundity, } \\
\text { decrease in egg hatching } \\
\text { success, teratogenic effects }\end{array}$ & $\begin{array}{l}\text { Inhibition of cell division: } \\
\text { microtubule } \\
\text { depolymerisation }\end{array}$ & $\begin{array}{l}\text { Miralto et al. (1999) } \\
\text { Ianora, Poulet \& } \\
\text { Miralto (2003) }\end{array}$ \\
\hline Eicosapentanoic acid & 'Diatom biofilms' & Invertebrates & Death & - & Jüttner (2001) \\
\hline \multirow[t]{3}{*}{ Fischerellin A } & \multirow[t]{3}{*}{ Fischerella muscicola } & Fungi & Growth inhibition & - & Hagmann \& Jüttner (1996) \\
\hline & & Micro-algae & Growth inhibition & PSI inhibition & Gross et al. (1991) \\
\hline & & Invertebrates & death & - & Hagmann \& Jüttner (1996) \\
\hline 12-epi-hapalindole E & Fischerella sp. & Bacteria and fungi & Growth inhibition & Inhibition of RNA synthesis & Doan et al. (2000) \\
\hline Nostocine A & Nostoc spongiaeforme & Micro-algae & & Formation of ROS & Hirata et al. (2003) \\
\hline \multirow[t]{2}{*}{ Nostocyclamide } & \multirow[t]{2}{*}{ Nostoc sp. } & Invertebrates & Death & - & Todorova \& Jüttner (1996) \\
\hline & & Micro-algae & Growth inhibition & - & Todorova \& Jüttner (1996) \\
\hline \multicolumn{6}{|l|}{ Toxins } \\
\hline \multirow[t]{4}{*}{ Anatoxin-a } & \multirow{4}{*}{$\begin{array}{l}\text { Cyanobacteria belonging } \\
\text { to the genera Anabaena, } \\
\text { Microcystis and Oscillatoria }\end{array}$} & Vertebrates & Muscles paralysis & $\begin{array}{l}\text { binds to nicotinic } \\
\text { acetylcholine receptor }\end{array}$ & \\
\hline & & Invertebrates & Decrease in fecundity & & $\begin{array}{l}\text { Gilbert (1994); Claska } \\
\text { \& Gilbert (1998) }\end{array}$ \\
\hline & & Aquatic plants & $\begin{array}{l}\text { Growth inhibition, reduction } \\
\text { in photosynthetic oxygen } \\
\text { production }\end{array}$ & Formation of ROS? & Mitrovic et al. (2004) \\
\hline & & Micro-algae & Cellular paralysis & - & Kearns \& Hunter (2001) \\
\hline \multirow[t]{3}{*}{ Cylindrospermopsin } & \multirow{3}{*}{$\begin{array}{l}\text { Aphanizomenon ovalisporum, } \\
\text { Cylindrospermopsis raciborskii, } \\
\text { Raphidiopsis curvata, } \\
\text { Umezakia natans }\end{array}$} & Vertebrates & $\begin{array}{l}\text { Heart failure, specific injuries } \\
\text { in different organs }\end{array}$ & $\begin{array}{l}\text { interference with protein/ } \\
\text { enzyme synthesis }\end{array}$ & Griffiths \& Saker (2003) \\
\hline & & Invertebrates & reduced fitness and growth & - & Nogueira et al. (2004) \\
\hline & & Terrestrial plants & $\begin{array}{l}\text { Growth inhibition, metabolism } \\
\text { inhibition }\end{array}$ & - & Vasas et al. (2002) \\
\hline \multirow[t]{4}{*}{ Microcystins } & \multirow{4}{*}{$\begin{array}{l}\text { Cyanobacteria belonging to } \\
\text { the genera Anabaena, Microcystis, } \\
\text { Oscillatoria and Planktothrix }\end{array}$} & Vertebrates & $\begin{array}{l}\text { Diarrhoea, vomiting, piloerection, } \\
\text { chronic inflammation of liver }\end{array}$ & $\begin{array}{l}\text { Inhibition of protein } \\
\text { phosphatases } 1 \text { and } 2 \mathrm{~A}\end{array}$ & Briand et al. (2003) \\
\hline & & Invertebrates & Death & - & Wiegand et al. (2002) \\
\hline & & Aquatic plants & $\begin{array}{l}\text { Growth inhibition, reduction in } \\
\text { photosynthetic oxygen production }\end{array}$ & - & $\begin{array}{l}\text { Pflugmacher }(2002,2004, \\
\text { Mitrovic et al. (2005) }\end{array}$ \\
\hline & & Micro-algae & Growth inhibition, cell death & Formation of ROS & $\begin{array}{l}\text { Vardi et al. (1999); } \\
\text { Hu et al. (2005) }\end{array}$ \\
\hline Saxitoxins & $\begin{array}{l}\text { Aphanizomenon flos-aquae, } \\
\text { Anabaena circinal is, } \\
\text { Cylindrospermopsis raciborskii, } \\
\text { Lyngbia wollei, Planktothrix sp. }\end{array}$ & Vertebrates & Muscles paralysis, death & $\begin{array}{l}\text { Binds to site } 1 \text { of sodium } \\
\text { channel of the cell } \\
\text { membrane }\end{array}$ & Carmichael (1994) \\
\hline
\end{tabular}

Figure 2: Summary of identified allelopathic compounds produced by freshwater algae. Effects and mode of action was included for select compounds (Leflaive, 2007). 


\subsection{Algal Media}

The basic requirements for high-density photoautotrophic microalgal cultures in enclosed photobioreactors are a powerful light source and proper distribution of light, efficient gas exchange, and suitable medium composition (Mandalam R., 1998). Specifically, algae require macronutrients, which include the following: carbon, nitrogen, phosphorus, iron, and magnesium. Along with these main nutrients, algae also require a diverse group of micronutrients and trace metals. Typically for laboratory cultures of algae, the carbon source is provided through the introduction of carbon dioxide through either continuous or intermittent sparging of the culture.

As algae grow in the culture media, they deplete the nutrients at an exponential rate. This is proportional to the exponential growth observed after introduction of microalgae into a nutrient rich environment. Because of this, the algae can only survive as long as the nutrient content of the media is conducive for growth. Assuming that carbon is periodically added to the system through carbon dioxide supplementation, the cultures will most likely reach limitation either through depletion of a trace metal or the nitrogen source.

\subsection{Study Objectives}

Outlined research questions:

1. What abiotic factors can be controlled, and how can they be controlled?

2. What is the most effective methodology for conducting a regrowth potential study?

3. What components constitute an ideal media for algal growth experiments? 
4. Does recycling culture media influence the growth of the successive culture?

5. Will anaerobically digested algal biomass function as the main source of nutrients for algal growth? 


\subsection{MATERIALS AND METHODS}

The procedures for operation, experimentation, and data analysis are described in the following section.

\subsection{Algal Species}

Throughout the course of the research several algal strains were used to conduct the experiments. The origin of the strains included Ward's Science, the University of Texas Culture Collection Agency, and San Luis Obispo raceway wastewater treatment ponds.

In January 2013, a strain of Chlorella sp. was purchased from Ward's Science, in San Luis Obispo, California. This strain was purchased as a $30 \mathrm{~mL}$ sample intended for use in academic settings. At Ward's Science, the strains are maintained under fluorescent lights in Erlenmeyer flasks capped with foam stoppers. Cultures are maintained in suspension using a magnetic stir bar and an associated magnetic stir plate. Figure 3 shows a photomicrograph taken of Ward's Chlorella using the microscopy setup discussed in Section 3.4.7. On average, these cells were 10 micrometers in diameter and consistently presented as circular in shape. Generally they were present as individual cells, but, under stressed conditions, they would group together in small colonies resembling grape-like clusters. 
Two strains were purchased from the University of Texas Cultures Collection (UTEX). Both cultures were

transported on an agar slant of Modified Bold's Basal Medium with vitamins and soil extract present. The first strain, Chlorella vulgaris (UTEX \#312), was obtained on July 2, 2013. Attempts to scale the culture up were unsuccessful due to the culture being dead on arrival. The

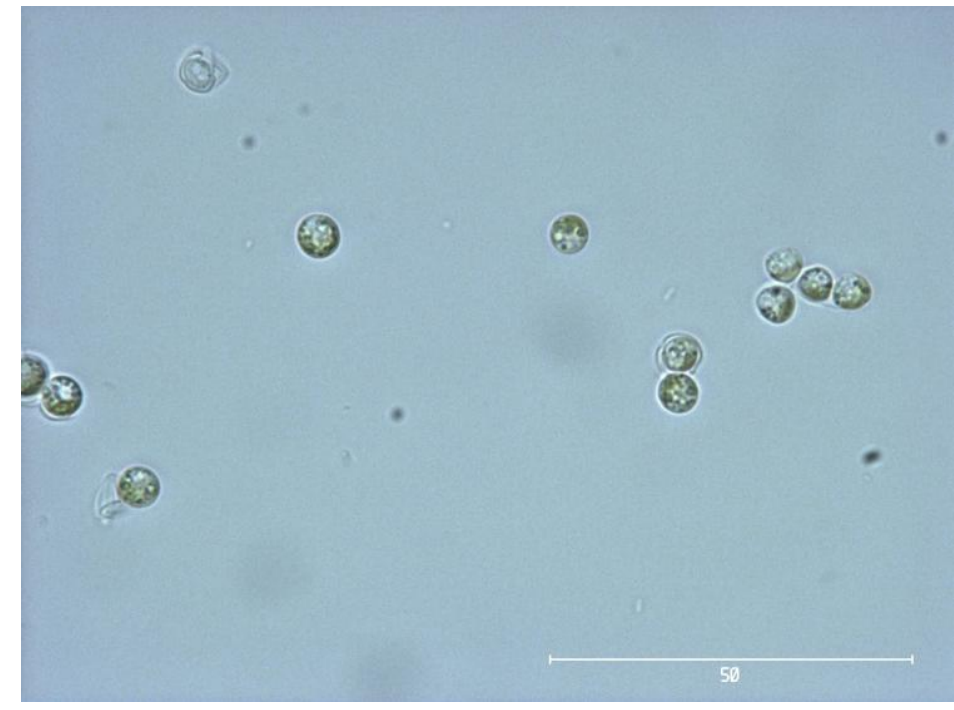

Figure 3: A photomicrograph showing Ward's Chlorella $s p$. The micrometer burnt into the image measures 50 micrometers. [1000x total magnification, brightfield] second strain, Scenedesmus dimorphus (UTEX \#1214), was obtained on August 20, 2013. This strain was successfully scaled up over the course of three weeks. However, during a malfunction of the incubator during December 2013 the majority of stock cultures were lost due to the resultant excessive temperatures inside of the

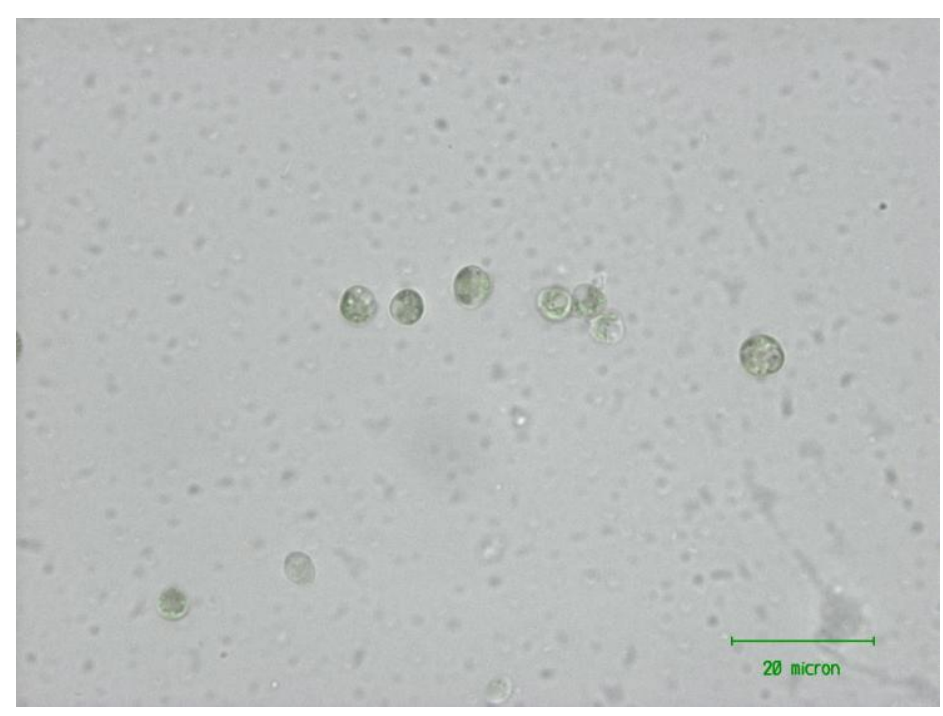
incubator. Several attempts were made to revive the cultures, however, the cultures were contaminated during this process and Figure 4: Photomicrograph of the isolated algal strain taken from wastewater treatment ponds in San Luis Obispo, CA. [400x total magnification, brightfield] were discarded. 


\subsubsection{Strain Isolation}

During the months of August through October a strain of algae was collected and isolated from the San Luis Obispo wastewater treatment plant. A grab sample was collected from the ponds and taken directly back to the laboratory. The sample was then serial diluted to factors ranging from $10^{-1}$ to $10^{-8}$ using Bold's Basal Medium and irradiated using a $23 \mathrm{~W}$ TCX SL desk lamp (Manufacturer \#: 35023-27K). The dilutions were allowed to grow for several days and then inspected under a microscope for uniformity. The most dilute sample that presented algal cells was then used as inoculum on an agar plate.

Plates were made using Bold's Basal Medium and 1.8\% laboratory grade agar. A standard isolation streak pattern was applied to the agar plate and then the plate was incubated at $28^{\circ} \mathrm{C}$ for three days. An individual colony was selected from the isolation plate and then reapplied to another agar plate using a streak isolation pattern again. A colony was then selected from this second agar plate and used to inoculate $5 \mathrm{~mL}$ of Bold's Basal Medium in a sterile $15 \mathrm{~mL}$ centrifuge tube. Figure 4 shows a photomicrograph of the final, isolated algal strain. The characteristics of the strain presented similar to Chlorella sp. with small clusters of circular cells with diameters between 5 and $10 \mu \mathrm{m}$.

\subsection{Media Preparation}

Bold's Basal Medium (Table 1) was selected as the primary media based on the previously described algal strains. This media was used by Ward's Science and the University of Texas Culture Collection Agency, UTEX, with Chlorella vulgaris. This media was originally created by Bischoff and Bold in1963 and has been extensively used as media for freshwater algae (Bischoff, 1963). 
Large quantities of this media were prepared on a regular basis to allow for the use of culture volumes ranging between $100-200 \mathrm{~mL}$ per bottle. To facilitate the creation of the media, stock solutions were made for each individual component of the media. The solutions were stored in capped, Pyrex bottles at ambient temperature $\left(\sim 22^{\circ} \mathrm{C}\right)$, and kept on a laboratory bench that did not receive direct sunlight. Each solution was remade in accordance to the use of the particular solution or after a maximum of one year of storage. Precipitation was only observed in the acidified iron trace metal solution, which was remade every approximately six months due to precipitation of the iron and adhesion to the Pyrex glass reagent storage bottle.

Along with these nutrients, sodium bicarbonate and several vitamins were incorporated into the base media recipe. Sodium bicarbonate was included in the above table but is not a traditional component of Bold's Basal Medium. These additions will be discussed later as they were a result of a comparative study in efforts to optimize the growth of the microalgae.

To avoid contamination, the medium was sterilized using one of two techniques: autoclaving or filtration. Each of these techniques had both benefits and drawbacks. One difficulty with autoclaving is the possibility of losing any compounds that can be offgassed.However, Bold's Basal Media contains no constituents that can be volatilized by autoclave sterilization. A Lancer Medical Services Autoclave (Serial No. 218718 was used to autoclave media and instruments used in the regrowth research. This method proved to be the most efficient as it allowed for large volumes (1-10 L) to be sterilized at the same time. 
Table 1: Nutrient constituents of Bold's Basal Medium for both 1N1P and 3N3P. 1N1P denotes one times the nitrogen and phosphorus content traditionally found in BBM media whereas 3N3P denotes three times the normal molar concentrations. Each constituent was stored in a separate, capped Pyrex bottle. Each constituent was added in the order listed when preparing the media. (NCMA Bold's Basal Medium) The volumes of each stock solution which were added to create one liter of Bold's Basal Medium were also included.

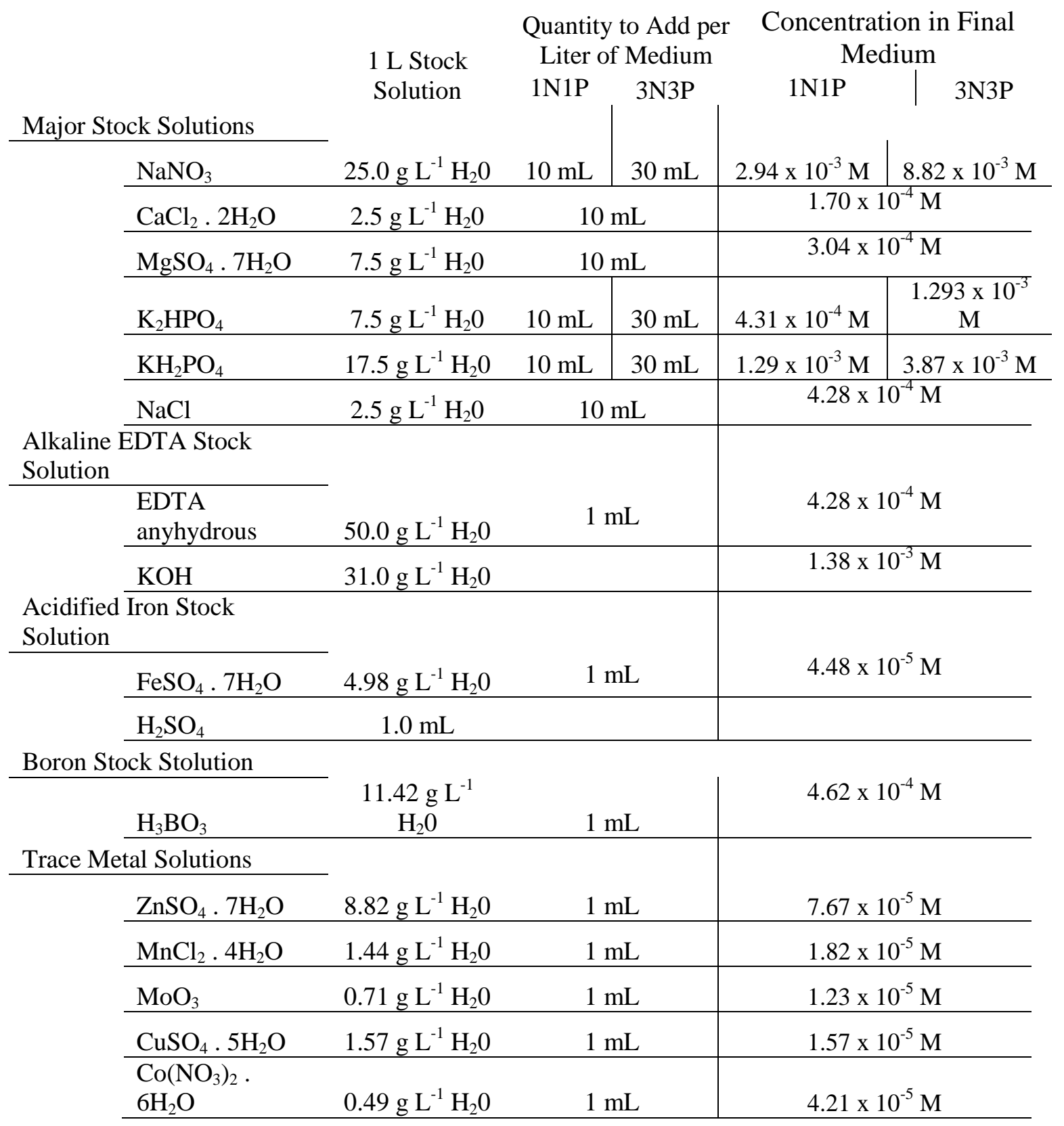


Any evaporative losses that occurred through the autoclave processes were replaced using sterile DI water. This method of sterilization raises the temperature of the entire media vessel to greater than $200{ }^{\circ} \mathrm{F}$. Therefore, it becomes essential to allow for the media to cool to ambient temperatures prior to inoculation with algae. Otherwise the excessive heat energy will pasteurize the inoculum resulting in an ineffective inoculation.

The filtration technique was necessary to allow for the introduction of both sodium bicarbonate and the vitamin solution into Bold's Basal Medium. Sodium bicarbonate was not autoclaved to avoid off-gassing of dissolved carbon dioxide. The vitamin solution was protected from high light and filter sterilized, not autoclaved, to prevent destruction of the vitamins. The three vitamins solution was stored in $15 \mathrm{~mL}$ centrifuge tubes in a freezer at $0{ }^{\circ} \mathrm{C}$. Filter sterilization was performed using a $0.22 \mu \mathrm{m}$ syringe filters (Millex GP Product \#: SLGP033RS) attached to 60-mL sterile dispensable syringes (Henke Sass Wolf Product\#: 12C088B).

Table 2: Vitamin concentrations of the modified Bold's Basal Medium used after January 2013. Each vitamin stock solution was prepared with HEPES buffer and adjusted to $\mathrm{pH}$ 7.8. Vitamins were stored in the freezer to preserve the vitamins and protect from heat and light degradation.

\section{Molar Concentration in Final}

Medium

\begin{tabular}{|c|c|}
\hline Vitamin Solutions & \\
\hline & \\
\hline vitamm B 12, & \\
\hline $\begin{array}{l}\text { Cyanocobalamin (Sigma V- } \\
6629 \text { ) }\end{array}$ & $9.953 \times 10^{-8} \mathrm{M}$ \\
\hline Biotin (Sigma B-4639) & $1.023 \times 10^{-7} \mathrm{M}$ \\
\hline $\begin{array}{l}\text { Thiamine HCL } \\
\text { (Sigma T-1270) }\end{array}$ & $1.113 \times 10^{-6} \mathrm{M}$ \\
\hline
\end{tabular}




\subsection{Growth Assay}

Sterile cultures were grown in Pyrex glass serum bottles (Fisher Catalog No. 06-451-42, Wheaton No. 223950). The serum bottles were sealed using natural silicon septa (Wheaton Product \#: 224174) and crimp aluminum caps (Wheaton Product \#: 22419701). The headspace was initially filled with pure carbon dioxide. Cultures were maintained in suspension through agitation via octagonal magnetic stir bars with a pivot ring (3.8 cm x $0.7 \mathrm{~cm}$ Fisherbrand Catalog No. S68070) and Thermo Scientific Cimarec Multipoint 15 Stir Plates (Catalog No. 50093538) (Figure5).

The culture volume was $200 \mathrm{~mL}$ with a headspace of $80 \mathrm{~mL}$. A larger culture volume allowed for maximization of the volume of media recycled between regrowth rounds. This was an important point in the growth assay as this also minimized the introduction of fresh culture media between rounds of growth. Stock cultures contained 100 to 150 $\mathrm{mL}$ of media. The larger headspace required less frequent carbon dioxide additions. The methodology for the addition of carbon dioxide to the algal culture is discussed further in Section 3.4.6 Carbon Additions. 
Continuous $\sim 250 \mu \mathrm{mol} / \mathrm{m}^{2}$-sec from $6400 \mathrm{~K}$ color temperature fluorescent bulbs.

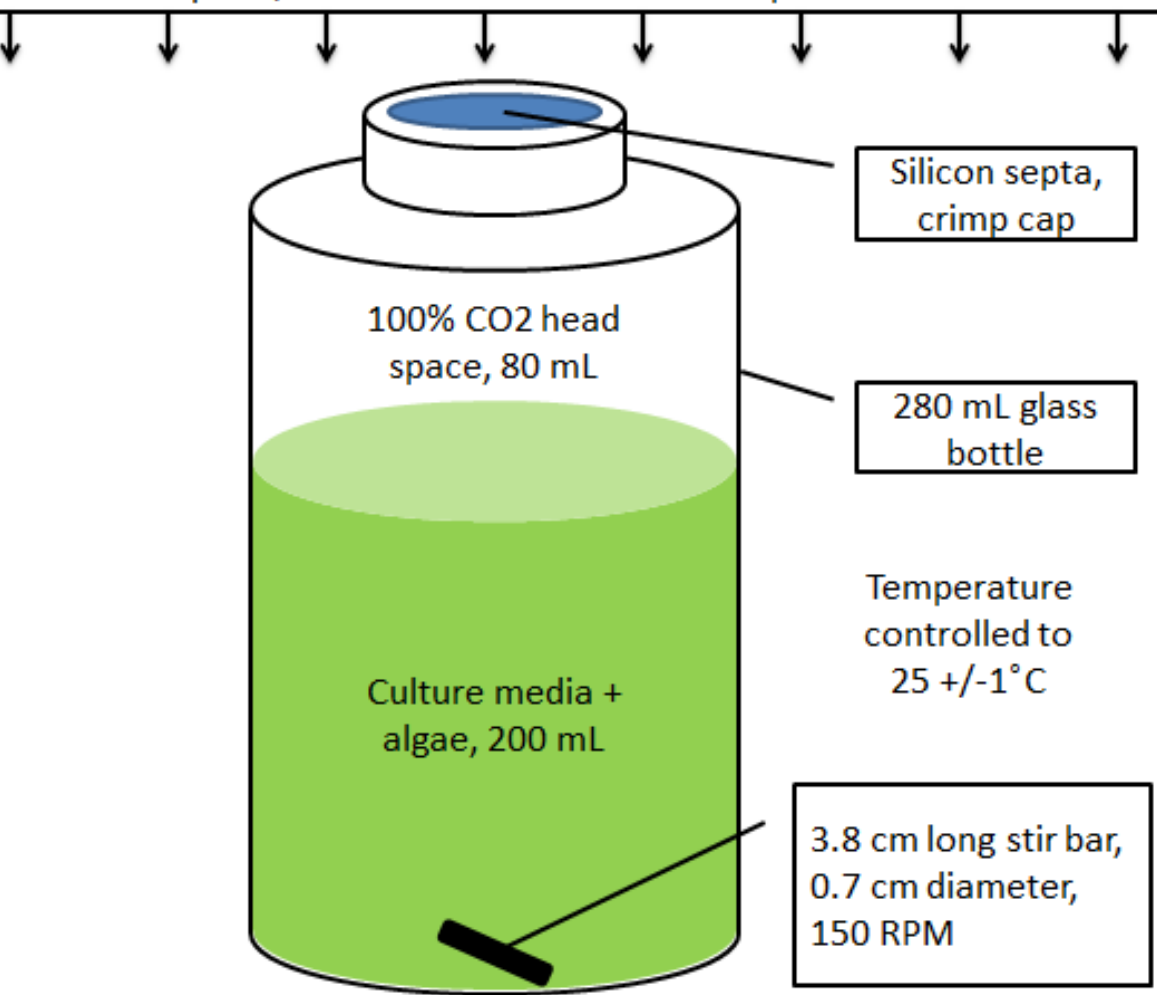

Figure 5: Graphic of the algae growth vessels. A pyrex glass bottle was sealed with a silcon septa and an aluminum crimp cap. Experimental culture volume was $200 \mathrm{~mL}$ with the remaining $80 \mathrm{~mL}$ composed of pure carbon dioxide.

\subsubsection{Lighting Conditions}

Algae are phototrophs and therefore require light as a primary component of metabolism. Under normal conditions the sun illuminates the earth with approximately $2000 \mu \mathrm{E}$ of lighting intensity at the surface. The algae use only a fraction of that light intensity during photophosphorylation to produce oxygen and energy from water. In order to maximize growth within the experimental cultures it was necessary to ensure the cultures were receiving near-saturation light intensity. Saturating lighting conditions allow for maximal growth rates of the algae, as well as decreasing the variance in lighting intensity observed by individual cultures. Algae were grown in two separate locations: an incubator and a vertical light rack. In order to measure the amount of light reaching the 
cultures, a Li-COR Li-250A Sensing Meter and a Li-190SA Quantom Sensor were purchased from Li-COR.

The first location was in a Controlled Environments Limited (Conviron) incubator (Product \#: CMP4030). Two 55W light hoods were purchased from HTG Supply to achieve this desired lighting intensity (Light Fixture: Product \# GL-HTG-TEK4). These lights emitted sufficient light intensities to achieve saturating lighting conditions (Avg. $246 \mu \mathrm{E})$. However, due to the refractivity of the glass culture vessels, the light intensity on the algae was below saturation $(180 \mu \mathrm{E})$. Each lighting apparatus contained eight $\mathrm{T} 5$ bulbs that emitted light at 6400 Kelvin (Product \# LAM-AM54G). This range of frequencies of light was chosen because it peaks emission at 430, 550, and 615nm. This closely matches with green algae, specifically Chlorella vulgaris, which has peak absorbance at 430 and $674 \mathrm{~nm}$ and minimum absorbance at $545 \mathrm{~nm}$ (refer to section 3.4.5 Biomass Measurement for a graphic of Chlorella's absorbance spectrum).

Two of these apparatus were used to provide light for up to twenty algae cultures. The number of cultures was limited to ensure the cultures were not shading one another. A

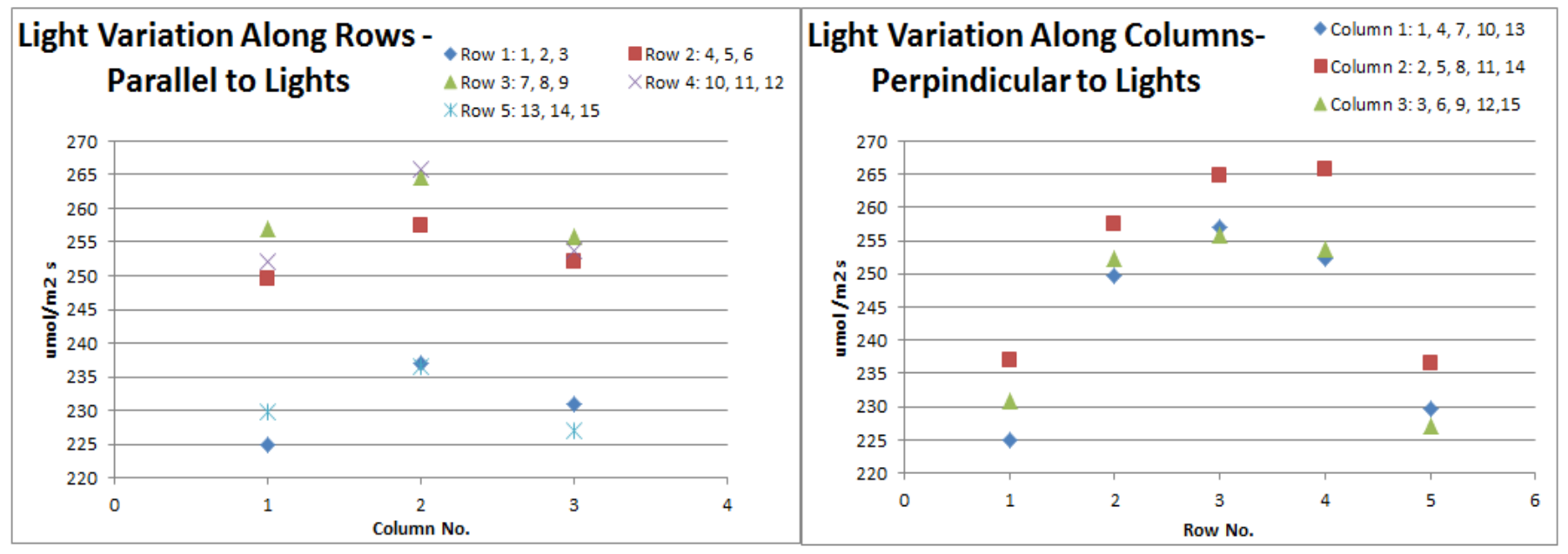

Figure 6: Light map generated for the incubator lighting array using the Li-COR sensing instrument. 
light map was created for the light hood and variance between the minimum and maximum intensity was determined to be less than $19 \%$. Although this variance was minimal, the cultures were rotated at random to further decrease the possible variance experience by a culture throughout the growth. Figure 6 shows the light map created for one of the two light apparatuses. The other lighting apparatus was also measured using the light sensor but was determined to differ less than 5\% from the measurements from the other light. Cultures were 1 foot away from the bottom of the light hood (measured to the liquid level).

The second location was in a laboratory, which utilized a lighting fixture created by Arizona State University. This fixture emitted light from ten fluorescent bulbs that were positioned vertically. The cultures were located approximately 2.5 inches away from the bulbs and provided approximately $280 \mu \mathrm{E}$ of light at the culture/column interface

\subsubsection{Temperature}

Temperature was maintained using the heating and cooling capabilities of the incubator. A target of $25^{\circ} \mathrm{C}$ was selected due to the high specific growth rates recorded for Chlorella at that temperature (Figure 7, Duata 1990). Furthermore, this temperature mimics the daytime temperatures experienced in San Luis Obispo, California. This allows data collected in the laboratory to be more easily applied and compared to data collected from the outdoor high-rate algae wastewater treatment ponds located at the San Luis Obispo Municipal Waste Water Treatment Facility. 
The second location, the light

rack, was not as temperature

controlled as the incubator.

However, the temperature of the

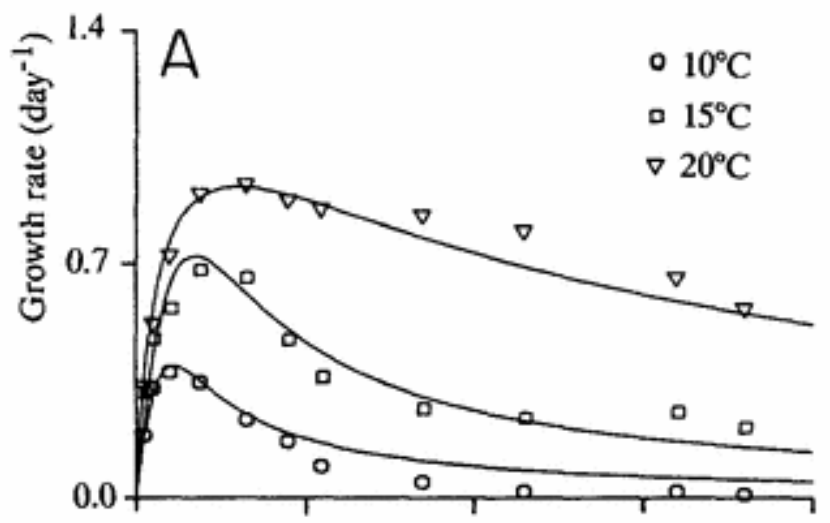

room was controlled by the

building's heating and cooling

system. The average temperature

was found to reside around $22{ }^{\circ} \mathrm{C}$.

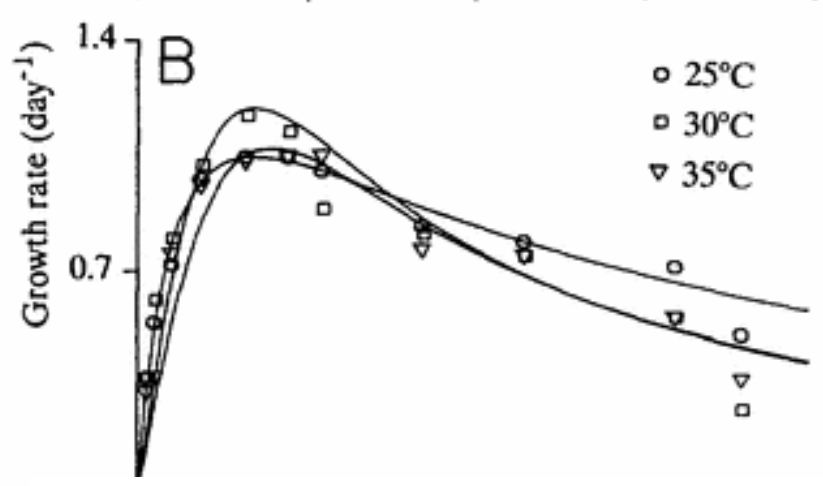

Figure 7: As temperature increases from 10-25 ${ }^{\circ} \mathrm{C}$ the growth rate of Chlorella vulgaris also increases. $25^{\circ} \mathrm{C}$ was chosen as the target temperature for this research due to high growth rates and mimicry of the ambient environment of San Luis Obispo, CA.

\subsubsection{Inoculation}

Target optical densities were calculated using the general dilution equation described below.

$$
V_{\text {Inoculum }}=\frac{V_{\text {Culture }} * O D_{\text {Culture }}}{O D_{\text {Inoculum }}}
$$

Where: $V_{\text {Inoculum }}$ refers to the volume of inoculum required to be transferred,

$\mathrm{V}_{\text {Culture }}$ refers to the volume of the new culture,

$\mathrm{OD}_{\text {Culture }}$ refers to the desired optical density of the new culture,

$\mathrm{OD}_{\text {Inoculum }}$ refers to the optical density of the culture being used as the inoculum 
Initial target optical densities ranged from 0.050 to 0.150 . This range of densities was chosen based on the detection limit of the spectrophotometer. As discussed section 3.4.5, Biomass Measurement, the calibration of the spectrophotometer demonstrated that optical densities below 0.050 were not consistent and therefore could not be used. A lower target optical density was used with experimental cultures to allow for more data points to be collected during the initial lag and exponential phases. In contrast, inoculations that utilize higher target optical densities will typically skip the initial lag phase and start directly in the exponential growth phase.

\subsubsection{Stock Culture Maintenance}

Stock cultures of each strain of algae were maintained in two separate locations for strain integrity protection. An orbital shaker was maintained under low lighting conditions of approximately $70 \mu \mathrm{E}$. This light intensity was far below the saturating lighting intensity for Chlorella and was intended to keep the algae at a depressed growth rate. Under this light intensity, the stock cultures were only transferred every few months as opposed to every five to seven days for experimental cultures. Sterility precautions were used when working with these preservation cultures as they were the only true backups for the algae strains.

The other stock cultures were maintained under a high light intensity of approximately $250 \mu \mathrm{E}$. This light intensity is near the saturating light intensity for Chlorella $s p$. and was provided by the same lights used for experiments. The purpose of these stock cultures was to maintain the algae in exponential growth. During this phase the algae rapidly reproduce and uptake nutrients making them effective during transfers. Transfers were

required every five to seven days to ensure the algae were not too dense or too old. Once 
the algae reached an optical density of around 1.4 the culture became opaque and selfshading. This is known as the light limited growth phase and significantly decreases the growth rates of the algae. Because the duration of the lag phase was not important for the stock cultures, they were inoculated to starting target optical densities of 0.100 to 0.150 . A higher starting optical density decreased the rate of unsuccessful inoculations and also initiated the growth of the algae allowing the culture to reach an optimal transfer optical density of 1.4 . 


\subsubsection{Biomass Measurement}

An indirect measurement of

biomass within the culture

was chosen to allow for

increased sampling

throughout the growth of the

algae. Optical density was

selected as the most

appropriate form of

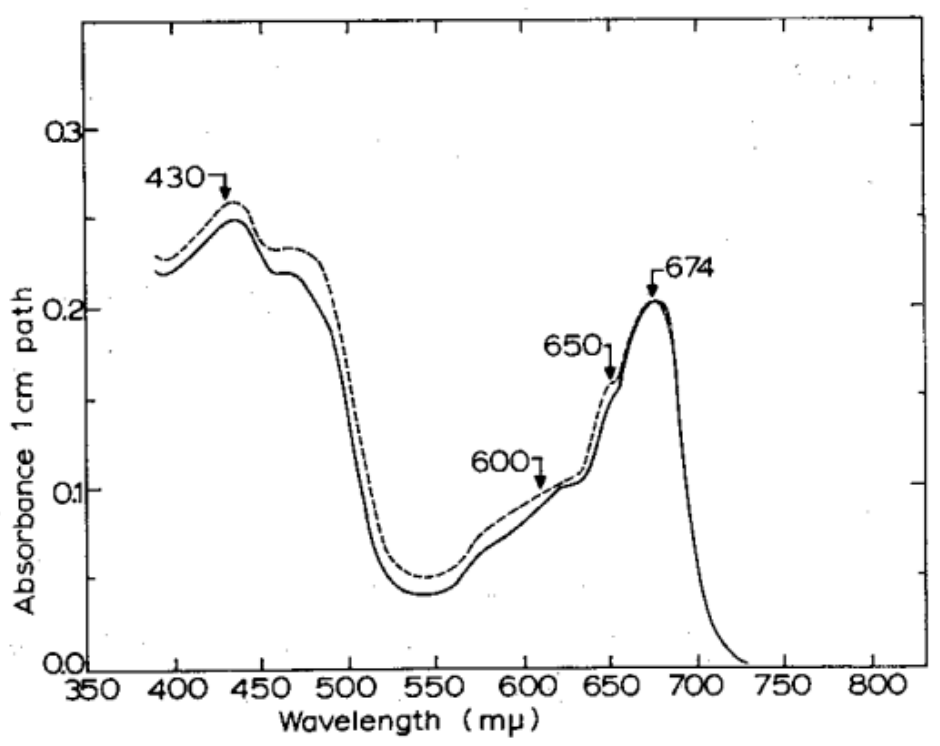

measurement due to the low

volume of sample required

Figure 8: The solid line depicts the absorbance spectrum of Chlorella vulgaris grown with water. The absorbance declines rapidly above a wavelength of $730 \mathrm{~nm}$.

(approximately $1 \mathrm{~mL}$ per

sampling event) as well as the low operational technicality of a spectrophotometer. Two spectrophotometers were used a Shimadzu UV-1700 PharmaSpec UV-VIS

Spectrophotometer (Serial \#: A110244) and a Specgenie 20 (Serial \#: 3SGA087099).

Algae can only absorb contain wavelengths of light. Absorption of light can be maximized by matching the absorbance spectrum for Chlorella vulgaris (Figure 8) with the emission spectrum of the light source. This graphic helps demonstrate why it was necessary, when measuring biomass, to choose a wavelength that the microalga species does not absorb. For this study $750 \mathrm{~nm}$ was selected due to Chlorella not absorbing light at this wavelength. 


\subsubsection{Carbon Additions}

Carbon dioxide was periodically purged into

culture vials to avoid carbon limitation. A

fifty-pound tank of industrial grade carbon

dioxide (Catalog No. UN1013) was

purchased from PRAXAIR. The carbon

dioxide was passed through a sterile 0.22

micrometer filter prior to entering the culture

vials to ensure foreign contaminates were

removed from the gas stream. A sterile

needle was then used to allow $100 \%$ carbon

dioxide to enter the culture vials while a

combination of oxygen and carbon dioxide

were vented from the cultures through a

secondary needle (Figure 9).

The purging time varied depending on the

relative $\mathrm{pH}$ of each culture vial. Typically,

cultures with a pH around $\mathrm{pH} 7.3$ were

purged for approximately 5 minutes, which

ensured the culture became saturated with

carbon dioxide. This resulted in a decrease

in $\mathrm{pH}$ to near 6.5.

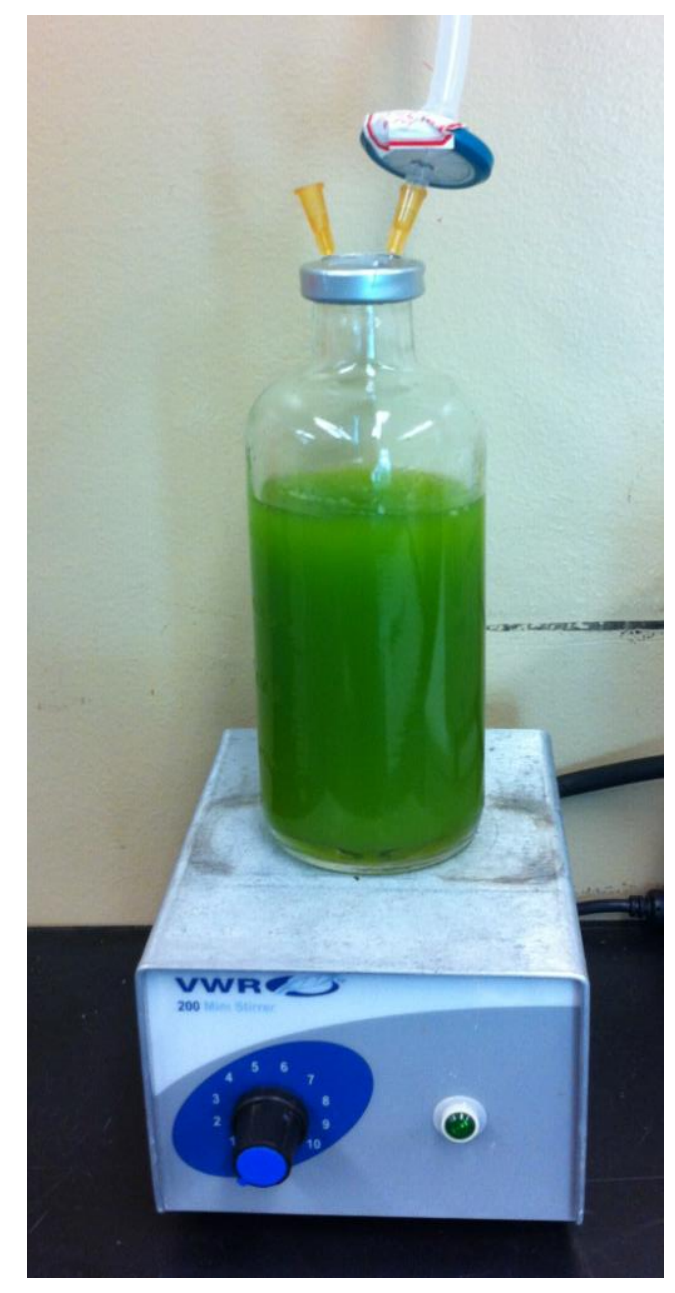

Figure 9: Carbon purging system for algae growth vessels. Culture was agitated using a stir plate while $100 \%$ $\mathrm{CO} 2$ was pumped into the headspace through the sterile syringe filter. An oxygen and carbon dioxide mixture exited the growth vessel through the second sterile needle. 


\subsubsection{Microscopy}

To maintain purity within each culture, periodic examinations were conducted using microscopy. The consistent size and morphology of Chlorella made it easy to confirm culture purity. Photomicrographs were taken at least once of every experimental culture in order to confirm strain integrity. Microscopy was also periodically conducted on the stock cultures. A micrometer scale was included with every photomicrograph to give size reference.

An Olympus CX 41 (Serial No. 6M10585 and 6M12454) microscope was used with an Olympus Infinity 2 Camera (Serial No. 7A21051 and 7A01304) to capture images of the samples. The Olympus program, Infinity Analyze, was used for image capturing and editing. . For this research both brightfield and phase contrast II were used with 100x and 400x total magnification, respectively.

\subsection{Recycled Media Experimental Design}

To demonstrate the possible existence of allelopathy within an algal culture a methodology was created to recycle the used media from each round of growth (Figure 10). Note that in the figure the blue vials represent new or restored media without algae, green vials represent media with algae, and yellow vials represent media in which a round of algae was grown or "used" media. In essence the regrowth experiment consisted of four main steps: the growth phase, harvest phase, nutrient restoration phase, and finally the reinoculation phase. 


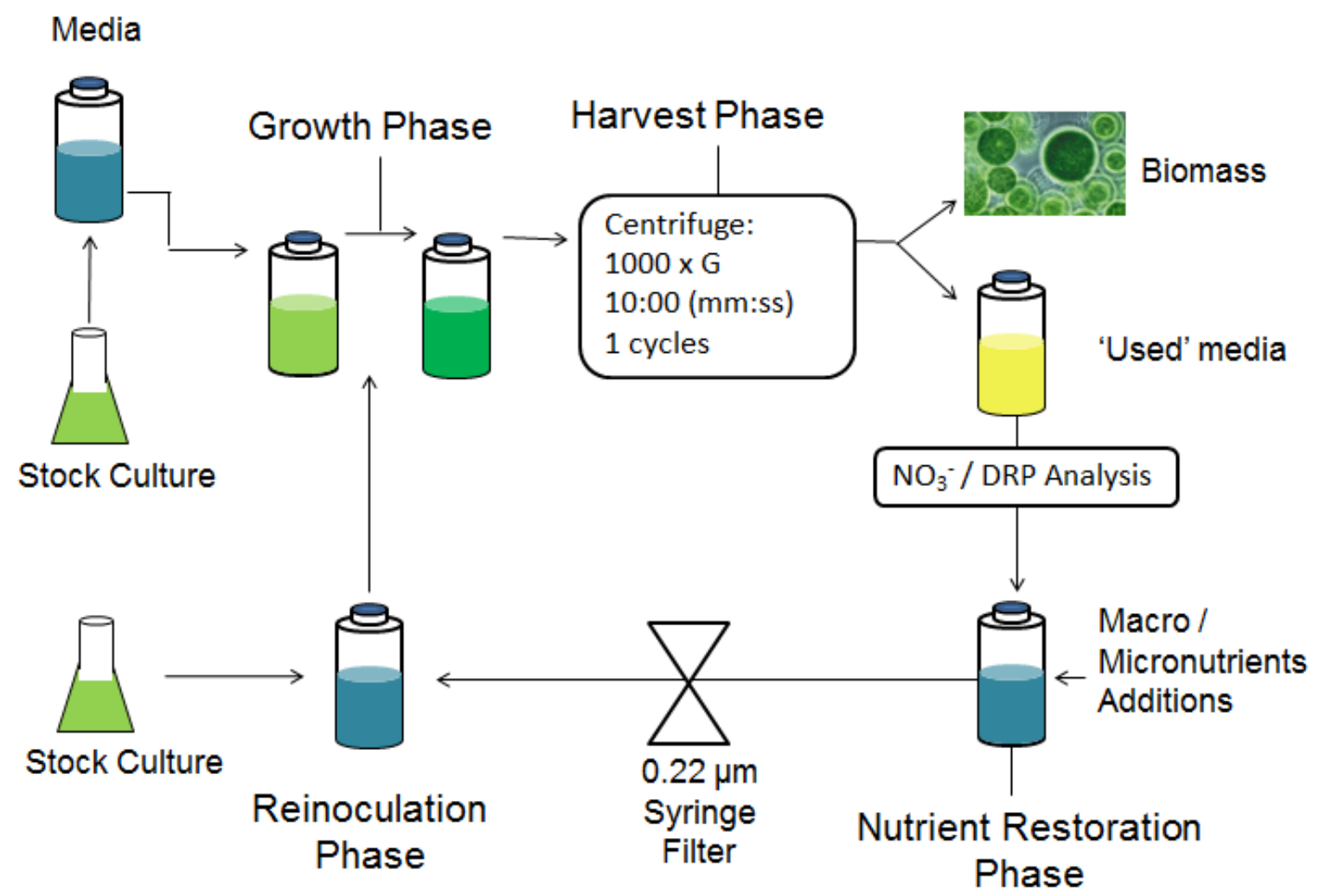

Figure 10: This figure shows a flowchart which describes the process of growing, harvesting, analyzing, restoring, and reinoculating for regrowth experiments.

Initially, algae was inoculated into fresh Bold's Basal Media and allowed to grow to midstationary phase. The culture was then combined with the other replicates and centrifuged using a Sorvall Legend XTR Centrifuge (Catalog No. 75004521, Serial No. 41291964). The centrifuge operated at 1000xg for ten minutes. By using high acceleration and a long spin duration, only one centrifuge cycle was necessary.

Once the biomass was partitioned, the sample was decanted into a vacuum filter apparatus. The apparatus consisted of a vacuum pump and a solids setup. Either a GAST Vacuum Pump (Model No. 1HAB-25b-M100X, Serial No. 0211011800 \& 0311001218), KNF Filtration Pump (Model No. UN816.1.2KT.45P, Serial No. 1/1348282), or a General Electric A-C Vacuum Pump (Model No. 5KH32EG550T, Serial No. 219500) 
was used to generate the vacuum pressure required for the solids extraction. The solids filtering apparatus consisted of a PyrexPlus Filtering Flask (Catalog No. 10-180K), a 47 mm Filter Holder (Catalog No. XX1004730), and a Bushnell Funnel.

Typically the centrifuged sample was filtered in several stages to reduce the solids loading onto the filter paper. First, the decanted supernatant of the centrifuged samples was passed through a G4 filter (Catalog No. 09-804-42C) stacked on top of a 0.45 micrometer filter (Catalog No. 09-719-2E). The G4 filter offered 1.1 micrometer filtration and acted as a pre-filter for the 0.45 micrometer filter. This filtration step removed all large cellular debris. After this preliminary filtration step, the nitrogen and phosphorus content of the "used" media was measured and restored to the initial concentrations outlined by the Bold's Basal Medium recipe. Finally, the restored media was sterilized using a sterile $0.22 \mu \mathrm{m}$ syringe filter with a $60 \mathrm{~mL}$ syringe,

\subsection{Nutrient Analysis}

The nutrient concentration of each culture was not periodically measured. This was determined impractical due to the volume required by many of the nutrient tests outlined by Standard Methods. This would decrease the active culture volume and change the conditions within the culture vessel quicker. For regrowth assays, it is important to allow the culture to reach mid stationary phase as this is the point in the microalgae's growth where it is hypothesized that allelopathic compounds are produced and released.

Instead, the nutrient concentration of cultures was measured at the end of each round of growth. This allowed for replenishment of the nutrients consumed throughout the growth. As nitrogen and phosphorus account for up to $9.7 \%$ of an algal cell's mass (on a 
dry basis) it was determined these would be the key nutrients to be measured and replenished after each successive round of growth (Mandalam R., 1998). Below is a more specific description of each the nutrient test, or tests, used to quantify both nitrogen and phosphorus.

\subsubsection{Nitrogen Analysis}

Algae are composed of 6-8\% (w/w) of nitrogen. In nature, algae may be exposed to nitrate, nitrite, ammonium, or ammonia as nitrogen sources. Throughout this research algae were grown on nitrate and ammonia.

A Timberline Instruments TL-2800 Ammonia Analyzer was used as the principle measurement of ammonia. A CETAC Technologies ASX-260 AutoSampler (Serial No. 121126A260) was added to the Timberline instrument to allow for automated sampling and measurement. This instrument first combines the sample stream with a caustic solution to raise the $\mathrm{pH}$ of the sample. At high $\mathrm{pH}$ values, the distribution of ammonia versus ammonium favors ammonium almost exclusively. After this conversion, the now caustic sample passes through a selective membrane through which the ammonium ions may permeate. A running buffer solution passes on the outside of the membrane and readily dissolves the ammonium ions. A conductivity sensor is located at the end of this process that measures the electrical conductivity of the buffer solution. As the ammonium permeates and combines with the buffer stream the conductivity increases. The electrical conductivity peak created by the additional ammonium present in the buffer is integrated. Samples should be filtered to a minimum of $0.45 \mu \mathrm{m}$ to ensure that the selective membrane does not become fouled with algal debris. 
The Timberline Ammonia Analyzer was also able to measure the nitrate content of samples. After the ammonia concentrations in the samples were measured, the samples were re-analyzed after a pre-treatment step. After combining with the caustic solution, the solution passed through a zinc reduction column. This reduction column converted any oxidized forms of nitrogen, primarily nitrate, into a reduced ammonium form. This additional ammonia in the sample increases the conductivity peak. The increased ammonia concentration is then subtracted from the ammonium ion measurement to yield the nitrate/nitrite concentration of the sample. It is important to note that a separate calibration curve was created for the nitrate analysis. Quality assurance and controls, including spikes and splits, were incorporated into the procedure to ensure accuracy and precision.

A second technique was used to measure nitrate exclusively. A nitrate probe (Serial No. RO1-14563) was used in conjunction with a Corning pH/Ion analyzer (Model 355, Serial No. C1467). The probe was calibrated with nitrate solutions with concentrations from 5 to 50 PPM.

\subsubsection{Phosphorus Analysis}

Mono and dibasic potassium phosphate were the only phosphorus constituents present in the Bold's Basal Medium. This form of phosphate can be readily utilized by the microalgae. An estimation of the bioavailable phosphorus in the culture media was made by analyzing the concentration of dissolved reactive phosphorus (DRP). This was measured in accordance with APHA Standard Methods 1995 Sections 4500- P A, B \& E. It was important to filter the sample through at least through a 0.45 micrometer filter to ensure that the photometric test was not skewed by the presence of cellular debris in the 
media. The calibration range for this test was between 0 and $1 \mathrm{mg} / \mathrm{L}$ as phosphorus. Therefore, it was necessary to dilute samples between 50 and 100x in order to stay within the calibration range.

\subsection{Nutrient Replenishment}

Using the data gathered from the analysis of both nitrogen and phosphorus, the percentage depletions of nutrients was calculated. The concentration of nutrients present in the media was compared to the Bold's Basil Media concentrations of nutrients and the volume of stock nutrient solutions to be added was calculated from the concentration of the nutrient stocks. The dilution required to return the total media to the initial volume was also taken into account. The lost volume arises from the periodic sampling, the nutrient analysis at the end of the experiment, and the minimal losses due to inefficiencies of the harvesting methodology. An example of the Excel spreadsheet used for the calculations is included in section 4.2.1 Media Carrying Capacity.

\subsection{Growth Kinetic Measurements}

The possible effect of allelopathy on growth of the experimental cultures was quantified using three separate variables: the duration of the lag phase, the exponential growth rate, and the light limited growth rate. Each of these variables refers to a specific section of a typical growth curve. Out of the four distinct regions (the lag phase, exponential growth phase, stationary phase, and death phase) these measurements span three regions (Figure11). These three growth kinetics will be further discussed in the subsequent sections. 


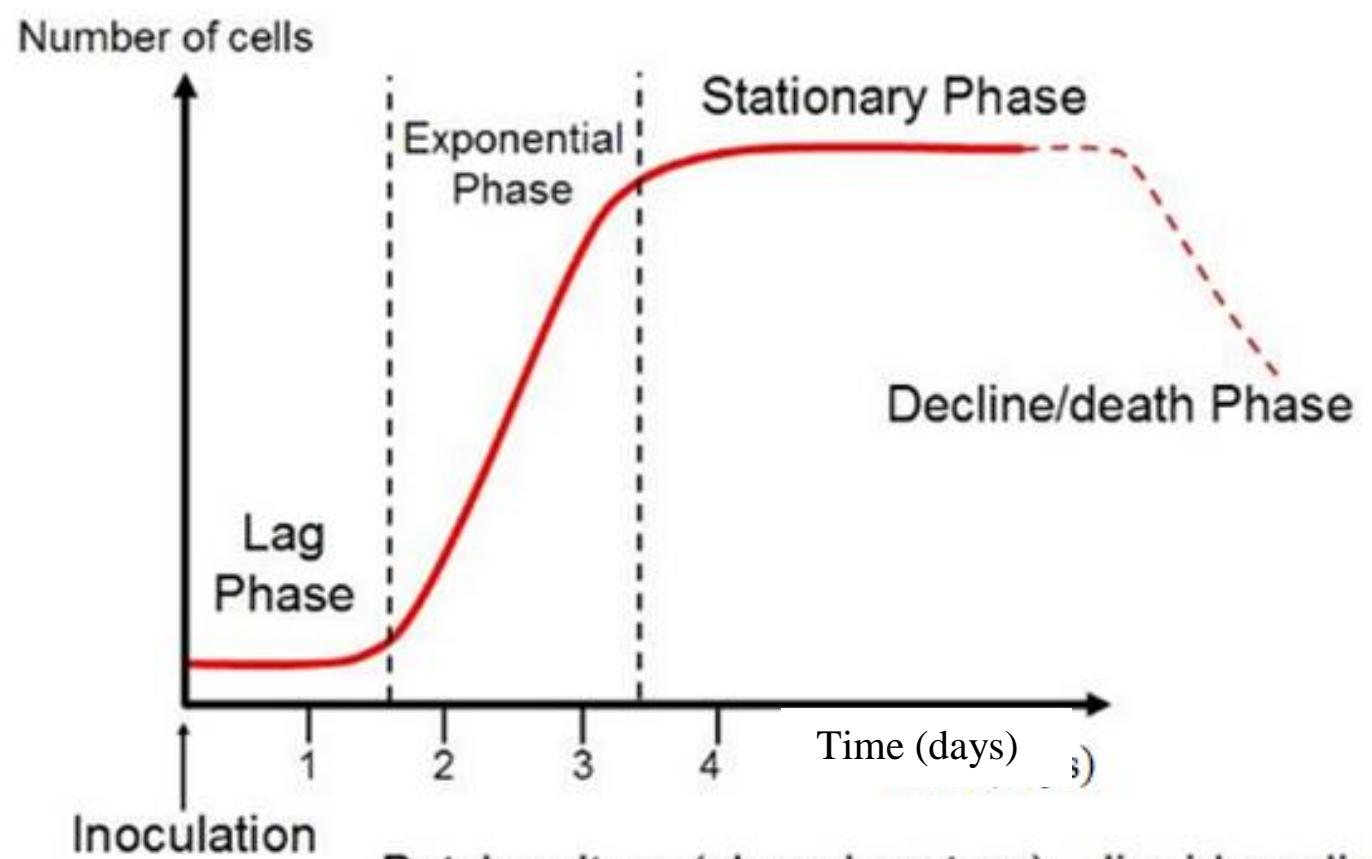

Batch culture (closed system) - liquid medium

Figure 11: Graphic depicting the classic growth curve for algae. For this research the main areas of focus included the lag, exponential, and stationary phase. This graphic does not depict the light limited growth region which occurs as the transition between the exponential and stationary phases. Adopted from Bacterial Growth and Regulation, 2007.

\subsubsection{Duration of Lag Phase}

After transfer into fresh media, algae typically require a short period of acclimation prior to replicating. This phase is termed the lag phase. On average, the duration of this phase is four to fifteen hours. Initially, the duration of this lag period was selected as a method for quantifying inhibition. However, as the researched progressed, it became apparent that the duration of the lag phase was sensitive to minute changes in inoculation.

Because of this large variance observed even between five replicates, it was necessary to avoid using the duration of the lag phase as a primary marker of inhibition. Instead, the duration of the lag phase was used to discern whether an inoculation was successful. 
Should the lag phase take longer than 15 hours, it was determined the inoculation was essentially ineffective and the cultures were restarted.

\subsubsection{Exponential Growth Rate}

During this phase, the optical densities of cultures were observed to increase by a factor of 20. Because of the rapid growth observed during this phase, samples were taken every four hours in order to obtain a minimum of three data points. This resulted improved the resolution of the growth curve and, therefore, the precision of the maximum specific growth rate. This also helped to differentiate between where the exponential growth region transitioned into the light limited growth region.

\subsubsection{Light Limited Growth Rate}

As algal cultures increase in density, the light intensity reaching through the culture decreases proportionally. Throughout the exponential growth phase the above saturating light intensity incident on the cultures is sufficient to penetrate the culture and allow for exponential growth. However, once the culture reaches a certain density, the incident saturating light can no longer penetrate the entirety of the algal culture. This point marks the beginning of the light limited growth region. This region presents linearly on a graph of optical density versus time. The growth rate determined from data collected in this region is termed light limited growth rate. The light limited growth rate is much less than the exponential growth rate. From this research, the light limited growth rate was determined to vary between one and twenty percent of the exponential growth rate recorded for the same culture. 


\subsection{Digestate Effluent Regrowth Experiments}

A growth experiment was also conducted using anaerobically digested algal biomass. During digestion of the biomass, hydrogen and methane are released which can be captured and used a fuel for heat or energy generation. After digestion the effluent is extremely rich in nutrients from the degradation of algal cells through thermogenesis and bacterial decomposition. The use of digester effluent for algal growth would further develop the feasibility of incorporating algal biomass as a biofuel due to the production of methane, hydrogen, and a nutrient-rich slurry, which can supplement algal cultures.

To prepare the digestate to be used in a growth study, the digester effluent was first filtered through at G4 filter that was stacked atop a 0.45 micrometer filter. Then, the filtrate was passed through a 0.22 micrometer filter which effectively sterilized the digestate. After filtration, the nutrient content of the digestate was analyzed using the DRP and Timberline Ammonia Analyzer described in Section 3.6.1. The digestate was kept in a sealed container to avoid volatilization of ammonia. However, the $\mathrm{pH}$ of the digestate was found to be below 7 where the equilibrium favors ammonium ion almost exclusively. Therefore, the risk of nitrogen loss from the system in the form of ammonia was minimal. The ammonia content was analyzed throughout the digestate growth experiment to determine if and when the culture became nutrient limited.

In contrast to the closed growth apparatus assay created for the regrowth experiments, the digestate growth experiment utilized a light rack. This growth apparatus was not temperature controlled; however, the building where the laboratory resides controls the ambient temperature in the building to approximately $22{ }^{\circ} \mathrm{C}$. Also, the columns were not sealed, but instead had a constant air and carbon dioxide gas mixture bubbling from the 
base of the column and escaping through a cotton-plugged vent hole in the stopper at the top. The culture volume for the columns was $200 \mathrm{~mL}$, which was dictated by the available digestate volume.

The experiment consisted of five controls and five digestate experiment columns. The controls contained all of the nutrients in Bold's Basal Medium in the listed concentrations of BBM3N3P except for nitrogen and phosphorus. These two nutrients were augmented to achieve concentrations equal to those present in the digestate. Due to the low volume of available sterilized digestate, it was necessary to dilute the digestate by $50 \%(\mathrm{v} / \mathrm{v})$. This allowed for the use of five replicates during the growth potential study. After the dilution, the nutrient content of the digestate was approximately $540 \mathrm{mg} / \mathrm{L}$ of ammonia as nitrogen, and approximately $650 \mathrm{mg} / \mathrm{L}$ of DRP as phosphorus. The digestate was diluted using a sterilized saline solution, which consisted of magnesium sulfate, sodium chloride, and calcium chloride. 


\subsection{RESULTS AND DISCUSSION}

This chapter discusses the results of the experiments outlined in the materials and methods chapter. Because of the nature of this project, the small experiments were organized into a graphical summary (Figure 12). This figure outlines which experiments were conducted and where they were included and discussed. All of the experiments conducted during this research either optimized the $\mathrm{pH}$ or the media, proved inhibition could be observed and quantified using the aforementioned methods, or was part of a complete regrowth experiment.

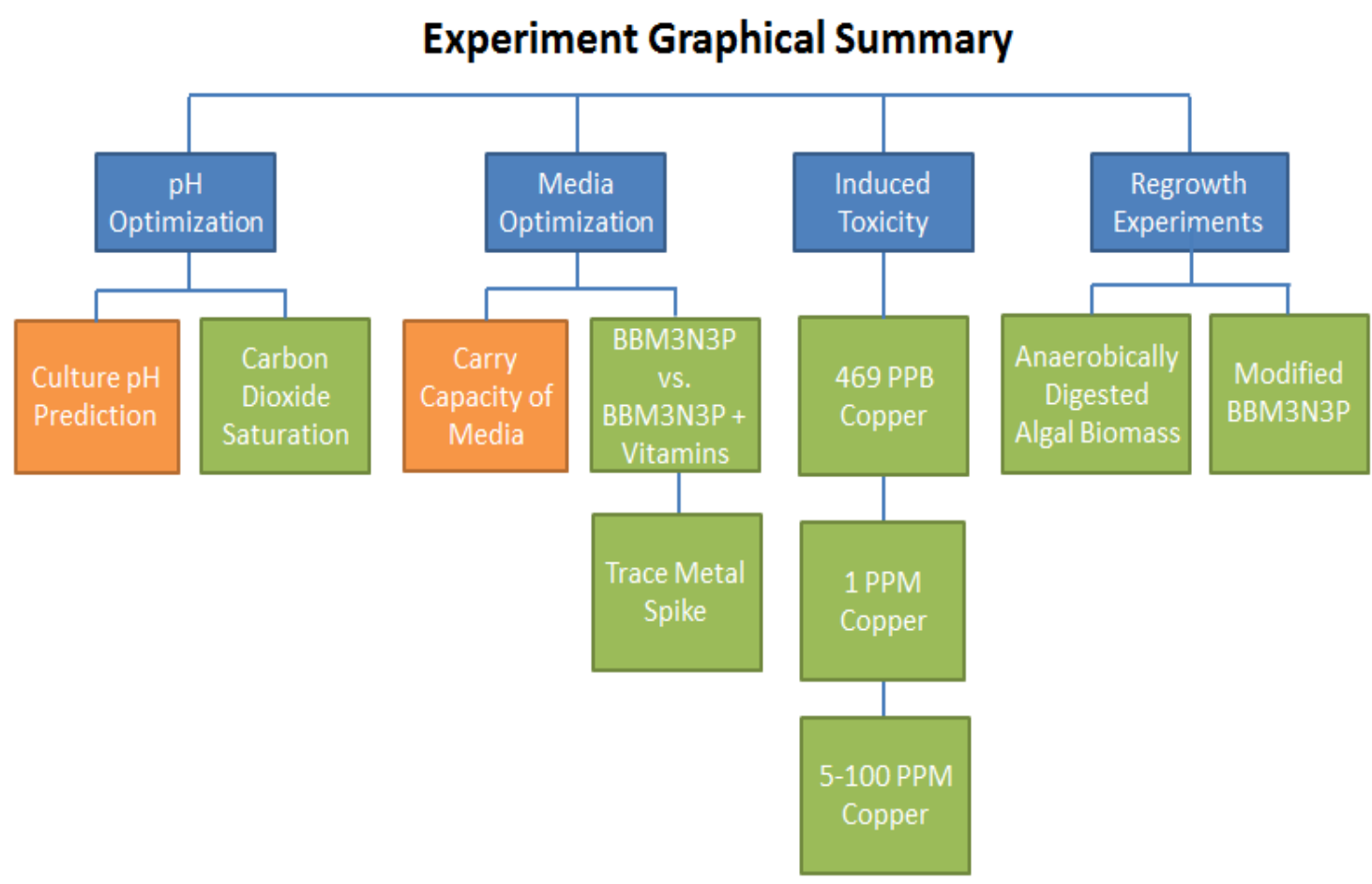

Figure 12: Flowchart describing the various experiments conducted to minimize the influence of external factors on growth. The orange boxes indicate calculations while the green boxes depict experiments that were conducted.

Experimental and theoretical optimization of the $\mathrm{pH}$ and media composition allowed for increased control over the abiotic factors of the research. The media optimization and trace metal/micronutrient spike experiments were designed to discern if any nutrient 
factors could be limiting the successive rounds of regrowth. Finally, a finalized regrowth was conducted with the optimized methodology and a growth potential experiment was conducted on digester effluent.

All experiments were inoculated from stock cultures, which were maintained in exponential growth. Growth rates of stock cultures were ensured to be above 0.80 doublings per day before being used to inoculate experimental cultures. This was done to warrant reproducibility between experiments as well as to ensure successful inoculations.

\section{1 pH Optimization}

Prior to initiating regrowth experiments, it was essential to evaluate and optimize the $\mathrm{pH}$ of the media. The growth assay for this research used sealed culture vials supplemented with $100 \%$ carbon dioxide. Thus, a pH buffer was needed. Bold's Basal Medium contains a phosphate buffer composed of mono- and di-basic potassium phosphate. However, the combined molar concentrations of the buffering system, $1.721 \times 10^{-3} \mathrm{M}$, could not buffer the pure carbon dioxide additions. Therefore, it was necessary to supplement the original Bold's Basal Medium with a stronger buffer. Sodium bicarbonate was chosen to act as the primary buffering compound because excessive phosphate addition might cause precipitation and some algae are able to use bicarbonate instead of $\mathrm{CO}_{2}$ as a carbon source.

\subsubsection{Culture pH Prediction}

Initially, a theoretical assessment was conducted to understand the amount of carbon dioxide required to support algal growth during an experiment (Kohn RA, 1998). 
Assuming that the culture volume was $200 \mathrm{~mL}$, the resulting headspace available for carbon dioxide was $80 \mathrm{~mL}$. Due to the low pressure of approximately one atmosphere, ambient temperature of $25^{\circ} \mathrm{C}$, it was further assumed that the ideal gas law would be acceptable for use in this application. Under complete dissolution of the carbon dioxide, the culture would contain $180 \mathrm{mg} / \mathrm{L}$ as carbon.

To estimate the additional sodium bicarbonate required to adequately buffer the media, the Henderson-Hasselbach equation was employed. This equation predicts the resulting $\mathrm{pH}$ of a solution containing a weak acid and its conjugate base. Noting that the acid dissociation constant for the equilibrium between carbonic acid and bicarbonate is $10^{-6.35}$ at $1 \mathrm{~atm}$ and $25^{\circ} \mathrm{C}$, a graph was created to determine the resulting $\mathrm{pH}$ for various bicarbonate concentrations (Figure 13). As seen in the figure below, a bicarbonate concentration between 10 and $100 \mathrm{mM}$ was required to produce a $\mathrm{pH}$ in the range of $5.75-6.75$

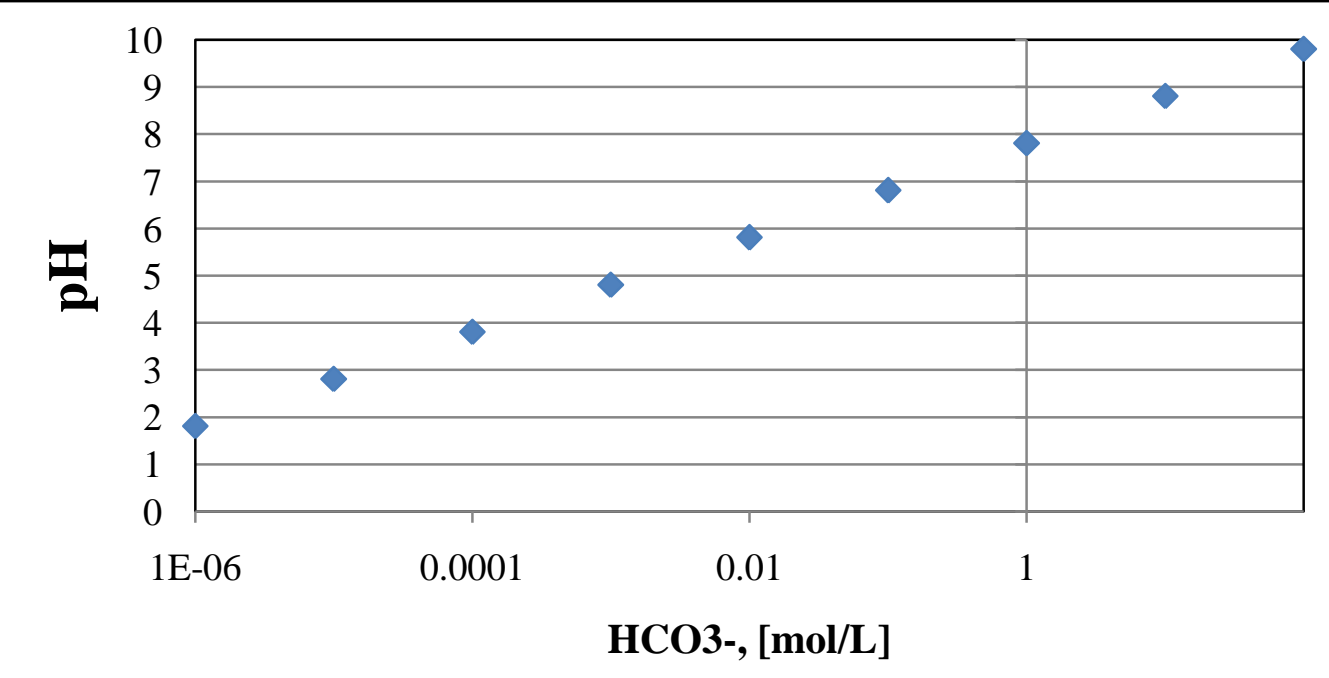

Figure 13: Predicted $\mathrm{pH}$ after $\mathrm{CO}_{2}$ saturation based on bicarbonate concentration 


\subsubsection{Carbon Dioxide Saturation Experiment}

An experiment was conducted to confirm the necessary concentration understanding that a bicarbonate concentration in the range of 1-100 $\mathrm{mM}$ would be required to keep the $\mathrm{pH}$ within viable limits for the algae. Sodium bicarbonate was added into Bold's Basal Medium in concentrations of 1,10 , and $100 \mathrm{mM}$. Each individual vial was purged with pure carbon dioxide for 5 minutes after which the resulting $\mathrm{pH}$ was recorded.

Originally, a pH of 6.5 was chosen as the minimum $\mathrm{pH}$ cut-off point, and a concentration of $100 \mathrm{mM}$ was required to achieve this $\mathrm{CO}_{2}$-saturated $\mathrm{pH}$ value (Figure 14). However, this concentration of bicarbonate was decided to be too high in comparison to the other constituents of Bold's Basal Medium. Therefore, $50 \mathrm{mM}$ was chosen to be the bicarbonate concentration introduced into the media. The resulting $\mathrm{CO}_{2}$-saturated $\mathrm{pH}$ value for the media was 6.25 , which would increase during growth.

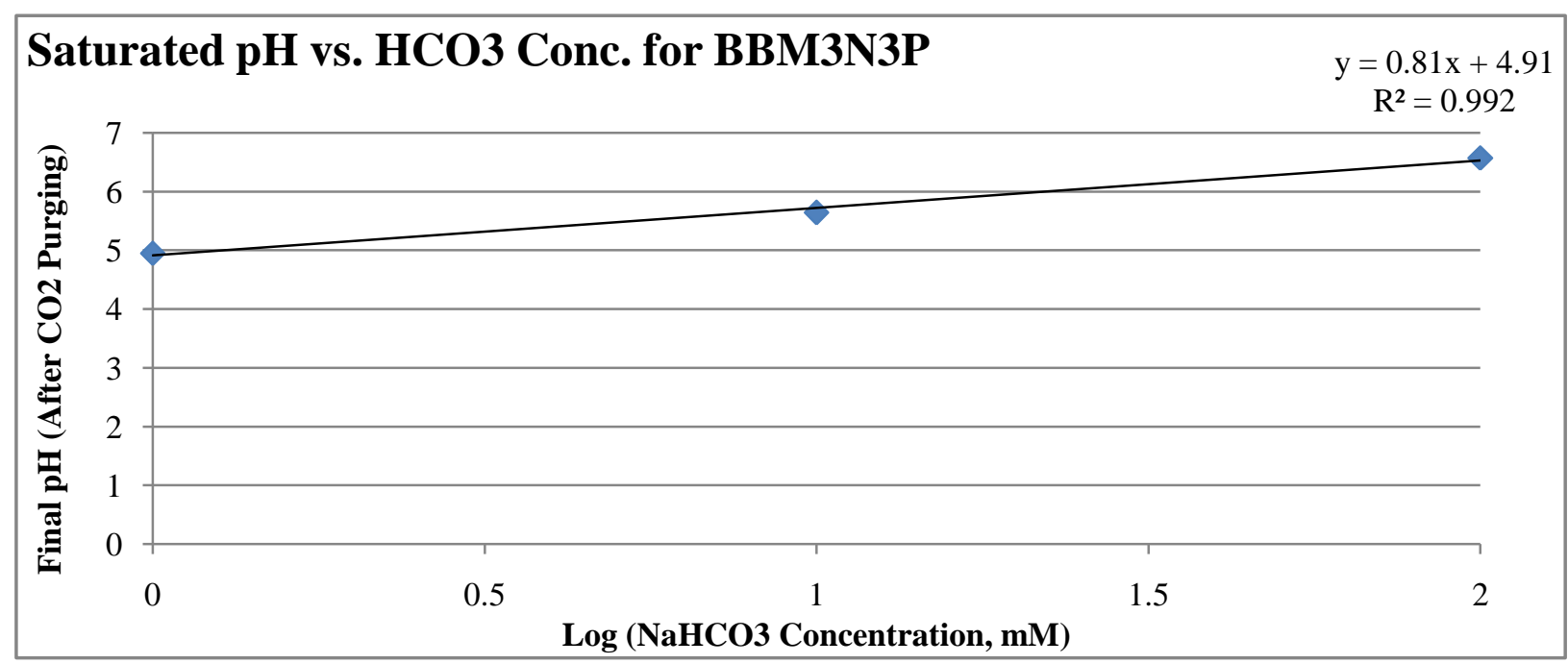

Figure 14: Graphic showing the resultant $\mathrm{pH}$ of the culture media after the introduction of various concentrations of sodium bicarbonate and saturation with gaseous carbon dioxide 


\subsection{Media Optimization}

Because the eventual goal of this research is to determine if inhibiting compounds are released into media during algae growth, it was essential to use a medium recipe that did not limit growth itself.

\subsubsection{Media Carrying Capacity}

The first step in the optimization of the media composition was a theoretical analysis of the carrying capacity of the media given its nutrient content. A spreadsheet was created within Microsoft Excel to aid in calculation of the maximum concentration of biomass each respective element within the media could support (Figure 15). Once the concentration was calculated, a rough estimate of the expected optical density was made using a correlation for Chlorella (pers. com. M. Huesemann, Pacific Northwest National Laboratory). This correlation allowed for the estimation of possible limiting nutrients within the algal media formulation. 


\begin{tabular}{|c|c|c|c|c|c|c|c|}
\hline \multirow{2}{*}{ Nutrient } & \multirow{2}{*}{$\begin{array}{l}\text { Conentration in } \\
\text { BBM 3N3P } \\
\text { culture media, } \\
{[\mathrm{mg} / \mathrm{L}]}\end{array}$} & \multicolumn{2}{|c|}{$\begin{array}{l}\text { Elemental composition } \\
\text { of Chlorella, [\% w/w }]^{*}\end{array}$} & \multicolumn{2}{|c|}{ BBM biomass carrying capacity, $\mathrm{mg} / \mathrm{L}$} & \multicolumn{2}{|c|}{$\begin{array}{c}\text { BBM biomass carrying capacity, OD, } \\
\text { using OD750 = AFDW/0.194 } \\
\text { (Huesemann 2013) }\end{array}$} \\
\hline & & $\begin{array}{l}\text { Low } \\
\text { Estimate }\end{array}$ & $\begin{array}{l}\text { High } \\
\text { Estimate }\end{array}$ & $\begin{array}{l}\text { BBM }[\mathrm{mg} / \mathrm{L}] \text { divided } \\
\text { by HIGH \% } \\
\text { composition }\end{array}$ & $\begin{array}{c}\text { BBM }[\mathrm{mg} / \mathrm{L}] \\
\text { divided by LOW } \\
\% \text { composition }\end{array}$ & $\begin{array}{l}\text { BBM }[\mathrm{mg} / \mathrm{L}] \\
\text { divided by HIGH \% } \\
\text { composition }\end{array}$ & $\begin{array}{l}\mathrm{BBM}[\mathrm{mg} / \mathrm{L}] \\
\text { divided by LOW \% } \\
\text { composition }\end{array}$ \\
\hline C & 180 & 45 & 48 & 375 & 400 & 1.9 & 2.1 \\
\hline $\mathrm{Fe}$ & 2.5 & 0.04 & 0.55 & 455 & 6255 & 2.3 & 32.2 \\
\hline $\mathrm{Mg}$ & 7.4 & 0.36 & 0.8 & 924 & 2052 & 6.9 & 13.6 \\
\hline $\mathrm{K}$ & 252 & 0.85 & 1.62 & 8524 & 16246 & 43.9 & 83.7 \\
\hline $\mathrm{S}$ & 14 & 0.28 & 0.39 & 3627 & 5053 & 18.7 & 26 \\
\hline $\mathrm{Ca}$ & 6.8 & 0.005 & 0.08 & 8516 & 136260 & 43.9 & 702.4 \\
\hline $\mathrm{Na}$ & 305 & - & - & - & - & - & - \\
\hline $\mathrm{Cl}$ & 29 & - & - & - & - & - & - \\
\hline B & 5.0 & - & - & - & - & - & - \\
\hline Mo & 1.2 & - & - & - & - & - & - \\
\hline Co & 0.2 & - & - & - & - & - & - \\
\hline
\end{tabular}

Figure 15: Graphic of the generated biomass carrying capacity spreadsheet used in analysis of nutrient limitation as well as nutrient restoration between rounds of regrowth experiments.

*Data from "Elemental balancing of biomass and medium composition enhances growth capacity in High-Density Chlorella vulgaris cultures" by Mandalam and Palsson. 
Standard Bold's Basal Medium contains $41.18 \mathrm{mg} / \mathrm{L}$ of nitrogen, which was insufficient for the desired cell densities in these experiments. The nitrogen content of the medium was therefore increased by a factor of three. By augmenting the nutrients by this factor, the cultures were able to reach light limitation prior to nutrient limitation. This allowed for quantification of the light limited growth rate of the algal cultures. Using the carrying capacity spreadsheet, it was estimated that the original Bold's Basal Medium could roughly support 375 to $400 \mathrm{mg} / \mathrm{L}$ of algal biomass, with carbon and iron being the limiting nutrients. This concentration correlates to an optical density of 1.9 to 2.1 . Comparing that to the modified Bold's Basal Medium with three times the nitrogen content, the media could support 536 to $666 \mathrm{mg} / \mathrm{L}$ of biomass or an optical density of 8.4 to 10.2. By having high biomass concentrations it was possible for the cultures to reach light limited growth. Further, these biomass concentrations are more comparable to a full-scale pond system which allowed for laboratory derived conclusions to be generally applied to full scale systems.

Along with inflating the nitrogen content, the phosphorus content was also scaled-up by a factor of three so that phosphorus would not be limiting relative to the added nitrogen and to have additional $\mathrm{pH}$ buffering. The ratio of mono- and di-basic potassium phosphate in Bold's Basal Medium was not changed during the scale-up in concentration in order to maintain the same buffered $\mathrm{pH}$ value as the original medium. Maintaining the buffer ratio became a priority during the nutrient restoration stage between regrowth trials. With the nitrogen and phosphorus contents increased, the only limiting nutrients indicated by the carrying capacity spreadsheet were carbon and iron. Carbon was added to the system throughout growth and therefore would never cause limitation. With a 
large range of Fe contents found in algae $(0.04$ and $0.55 \%$ w/w, Oh-hama and Miyachi, 1988), it was decided that iron would not likely become limiting during one round of growth.

\subsubsection{Vitamin Additions}

The vitamins cyanocobalamin $\left(\mathrm{B}_{12}\right)$, thiamine $\mathrm{HCl}$, and biotin were added to the original Bold's Basal Medium at the suggestion of culture maintenance personnel at University of Texas culture collection (UTEX). Before routinely augmenting the media recipe with vitamins, a comparative study was conducted between normal and vitamin-enhanced Bold's Basal Medium. All other components of the media, including bicarbonate, were identical in the control and vitamin-enhanced versions of BBM. Data from the experiment was plotted on both normal and logarithmic graphs of growth (Figure 16). Five replicates were included for both the control and the modified BBM media. The average maximum specific growth rates for the normal BBM and for the vitaminenhanced BBM were $0.689+/-0.0818$ and $0.887+/-0.1901$ (mean $+/$ - std. deviation) per day, respectively.

Although there was high variance observed throughout the experiment (12\% for BBM and $21 \%$ for Modified BBM with vitamins), a t-test determined there was a significant difference between the data sets with a p-value equal to 0.041 . Vitamins were then included in BBM because vitamins did not hinder the growth of the algal cultures. Further, prior experiments showed that sickly algal cultures could be revived by the addition of vitamin $B_{12}$. Sickly describes cultures that grew particularly slowly and/or had a yellowish color. 


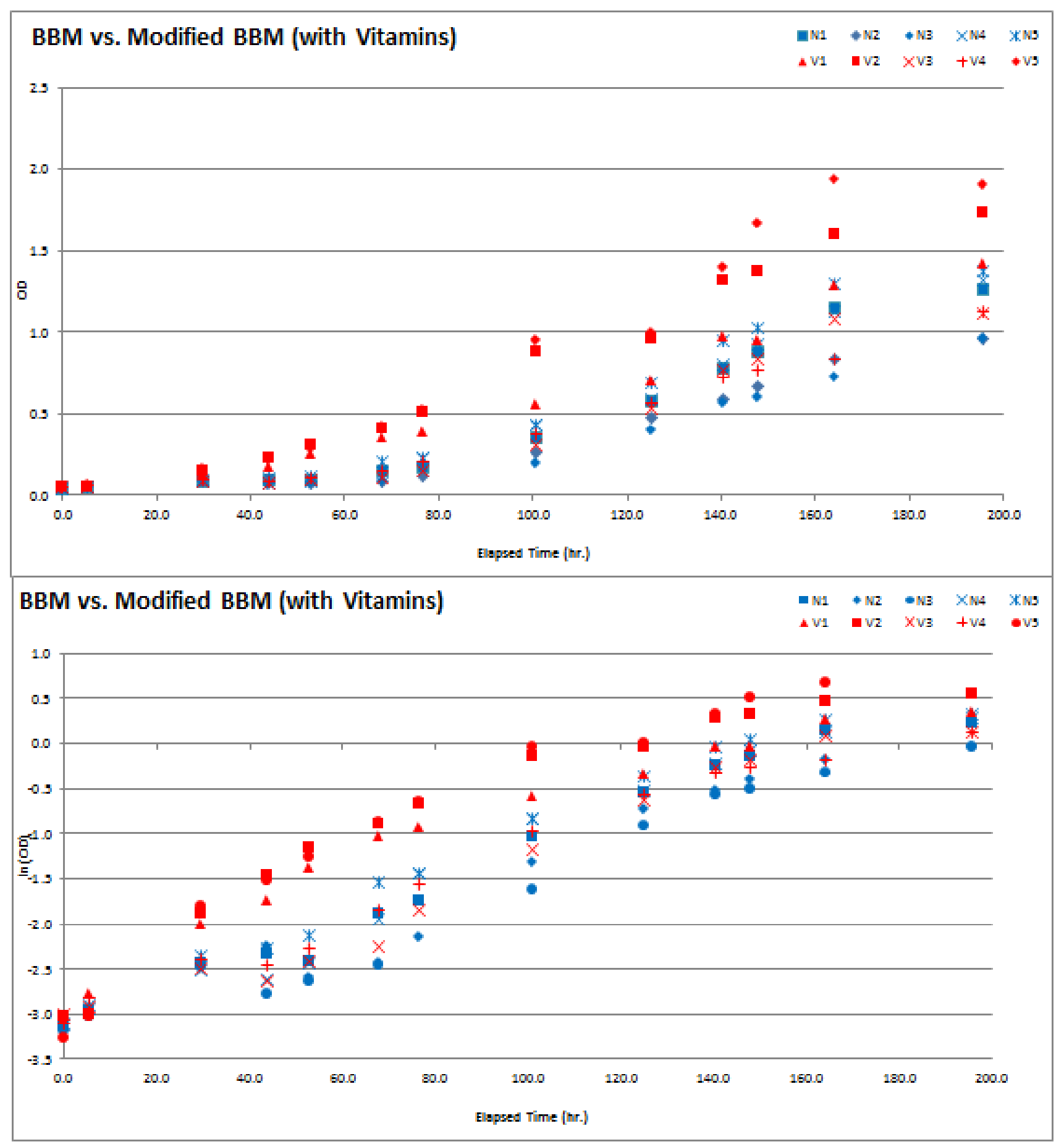

Figure 16: Plotted growth data from comparison study between normal and vitamin-enhanced Bold's Basal Medium. The top figure shows a normal OD vs. time, while the bottom figure shows the optical density on a logarithmic scale. $\mathrm{N}$ denotes the control cultures which contained the original BBM (1N1P) recipe while $\mathrm{V}$ denotes the cultures which had vitamins added. 


\subsection{Trace Metal and Micronutrient Spike}

Ensuring the cultures did not reach nutrient limitation was an important aspect of the project. However, some of these nutrients could become inhibitory at excessive concentrations. To test if the planned repeated trace metal additions would be inhibitory, a 4x4 spike experiment was conducted using Ward's Chlorella sp. Four controls were grown using Bold's Basal Medium modified to three times the nitrogen and phosphorus (3N3P) with the addition of vitamins and bicarbonate (concentrations outlined in section 3.2 Media Preparation). The test cultures were also made with the same media as the controls but had an additional seven times the micronutrients, specifically the alkaline EDTA, acidified iron, boron, and trace metals listed in media recipe for Bold's Basal Medium. Essentially, the test cultures were mimicking the eighth round of regrowth under the conservative assumption that no trace metals were consumed or removed during any of the previous seven rounds of growth. This assumption was made as a worst-case scenario to ensure restoring the micronutrients after each round of growth would not induce any inhibition.

Figure 17 shows the data collected during the trace metal spike experiment. Cultures denoted by "C" were controls and cultures denoted by " $\mathrm{T}$ " were the experimental treatment cultures. An analysis of Experiment 1 showed small relative standard deviation among the replicates (Table 3). Further, a student's t-test was performed on the controls and the experimental cultures. At a $\mathrm{p}$ value of 0.0956 , there was no significant difference at $95 \%$ confidence level. Because this test only used data collected during the exponential growth region, the first experiment was ended after the cultures reached light limitation. 


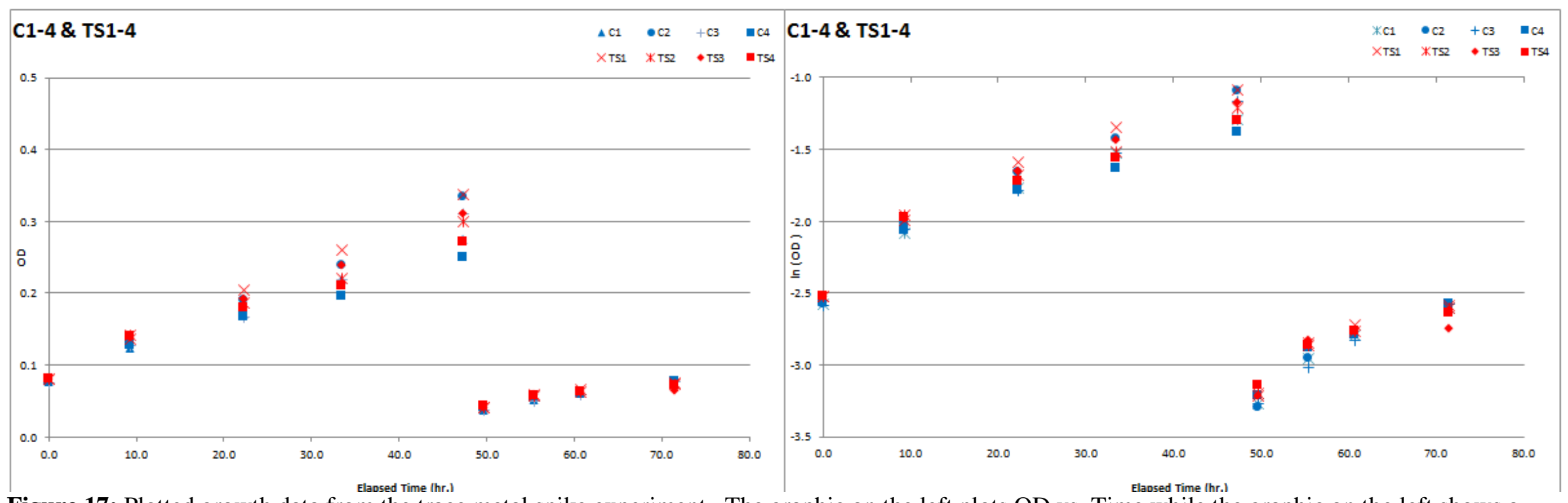

Figure 17: Plotted growth data from the trace metal spike experiment. The graphic on the left plots OD vs. Time while the graphic on the left shows a logarithmic plot of OD vs. Time. The experiment was restarted at a time equal to 50 hours.

Table 3: Data collected from the trace metal spike experiment. C1-4 refer to the control cultures, while T1-4 refer to the experimental cultures with 8x micronutrients. Experiment 1 refers to the plotted data from 0-50 hours. At that time, the cultures were discarded, the bottles refilled with fresh media, and reinoculated, marking the beginning of Experiment 2 which extended from hours 50-72.

\begin{tabular}{|c|c|c|c|c|c|c|c|c|c|c|c|c|c|c|c|c|}
\hline & \multicolumn{8}{|c|}{ Experiment 1} & \multicolumn{8}{|c|}{ Experiment 2} \\
\hline & $\mathrm{C} 1$ & $\mathrm{C} 2$ & $\mathrm{C} 3$ & $\mathrm{C} 4$ & TS1 & TS2 & TS3 & TS4 & $\mathrm{C} 1$ & $\mathrm{C} 2$ & $\mathrm{C} 3$ & $\mathrm{C} 4$ & TS1 & TS2 & TS3 & TS4 \\
\hline$\mu_{\max }\left(\right.$ day $\left.^{-1}\right)$ & 1.26 & 1.44 & 1.38 & 1.29 & 1.37 & 1.46 & 1.40 & 1.42 & 1.31 & 1.42 & 1.06 & 1.40 & 1.55 & 1.38 & 1.62 & 1.18 \\
\hline $\begin{array}{l}\text { Avg. } \mu_{\max } \\
\left(\text { day }^{-1}\right)\end{array}$ & \multicolumn{4}{|c|}{1.34} & \multicolumn{4}{|c|}{1.41} & \multicolumn{4}{|c|}{1.30} & \multicolumn{4}{|c|}{1.43} \\
\hline S.D. & \multicolumn{4}{|c|}{0.0831} & \multicolumn{4}{|c|}{0.0381} & \multicolumn{4}{|c|}{0.166} & \multicolumn{4}{|c|}{0.199} \\
\hline R.S.D. & \multicolumn{4}{|c|}{$6.2 \%$} & \multicolumn{4}{|c|}{$2.7 \%$} & \multicolumn{4}{|c|}{$12.8 \%$} & \multicolumn{4}{|c|}{$13.9 \%$} \\
\hline T-Test & \multicolumn{8}{|c|}{0.0956} & \multicolumn{8}{|c|}{0.174} \\
\hline
\end{tabular}


At 50 hours, the culture vessels were emptied, refreshed with new media, and inoculated with new Ward's Chlorella from the stock cultures. Data collected from Experiment 2 showed growth rates that were similar to Experiment 1. However, the deviation among the replicates was substantially higher during the second experiment with RSD values of $12.8 \%$ and $13.9 \%$ for the controls and experimental cultures, respectively. The t-test performed on this data set produced a $\mathrm{p}$ value equal to 0.1736 , which again indicates no significant difference existed between the controls and experimental cultures at a $95 \%$ confidence level.

\subsection{Induced Toxicity}

To improve confidence that the methods used did not result in false negatives for inhibition, tests were conducted to purposefully inhibit cultures. According to the “Guidelines for the Testing of Chemicals Freshwater Algal and Cyanobacterial Growth Inhibition Test" (OECD, 2006), a reference chemical such as potassium dichromate or 3, 5-dichlorophenol may be used to check the test algae growth procedures. For the present research, copper chloride was selected as reference compound due to its noted toxicity to algae.

The initial concentration of copper chloride was selected based on a literature review. According to Franklin (2002), a copper concentration of ten parts per billion (ppb) was required to achieve an EC50. (EC50 refers to a 50\% decrease in the 72-hour mean cell concentration as measured by a flow cytometer). Franklin used Chlorella sp. as the algal strain, which made the results applicable to the Ward's Chlorella. However, at the time of this experiment the only algal strain ready for transfer was ISO. Therefore, this strain was selected for this preliminary toxicity study. 
Copper chloride was first oven dried at $105^{\circ} \mathrm{C}$ for over 12 hours to remove all moisture and return the copper chloride to an anhydrous form. The initial concentration chosen for the experiment was $1 \mathrm{mg} \mathrm{L}^{-1}$. Therefore, the toxicant dose added to the algal cultures was $469 \mathrm{ppb}$, which is approximately 47 times more concentrated than the dose used in Franklin's research.

The first inhibition test was inoculated on October 28, 2013. Figure 18 shows the growth data collected from the experiment. Three controls, C1-3, and three experimental cultures, T1-3, were all inoculated from the same stock culture to a target optical density of 0.080. The inoculated densities ranged from 0.059-0.081.

The average maximum specific growth rates for the controls and test cultures were 1.247 and 1.034 per day, respectively. . A student's t-test with a p value equal to 0.0146 , confirmed that there was a significance difference between the exponential growth rates of the control and test cultures at the 95\% confidence level. Along with the t-test results, the relative standard error within the data for the controls and test cultures was $2.53 \%$ and $6.14 \%$, respectively, which shows congruency between replicates (Table 4). However, a visual inspection of the data suggests that the test culture $\mathrm{T} 3$ grew significantly slower than any other cultures. When this data were omitted from the analysis, there was no significant difference. However, this left only two experimental cultures contributing to the analysis. Due to the low sample size, the conclusion cannot be derived with significant confidence. Therefore, the test was repeated. 
Table 4: Data gather from $\mathrm{Cu}$ induced inhibition of the ISO strain. The $\mathrm{p}$ value obtained from the student $\mathrm{t}$-test confirmed that a significant difference exists between the control and test cultures. C1-3 refer to the control cultures while T1-3 refer to the test cultures.

\begin{tabular}{|c|c|c|c|c|c|c|}
\hline & \multicolumn{6}{|c|}{ Culture Identification } \\
\hline & C1 & $\mathrm{C} 2$ & C3 & T1 & $\mathrm{T} 2$ & T3 \\
\hline$\mu_{\max }\left(\operatorname{day}^{-1}\right)$ & 1.242 & 1.282 & 1.219 & 1.058 & 1.082 & 0.962 \\
\hline Avg. $\mu_{\max }\left(\right.$ day $\left.^{-1}\right)$ & \multicolumn{3}{|c|}{1.25} & \multicolumn{3}{|c|}{1.03} \\
\hline S.D. & \multicolumn{3}{|c|}{0.03162} & \multicolumn{3}{|c|}{0.06350} \\
\hline R.S.D. & \multicolumn{3}{|c|}{$2.5 \%$} & \multicolumn{3}{|c|}{$6.1 \%$} \\
\hline T-Test & \multicolumn{6}{|c|}{0.00729} \\
\hline
\end{tabular}

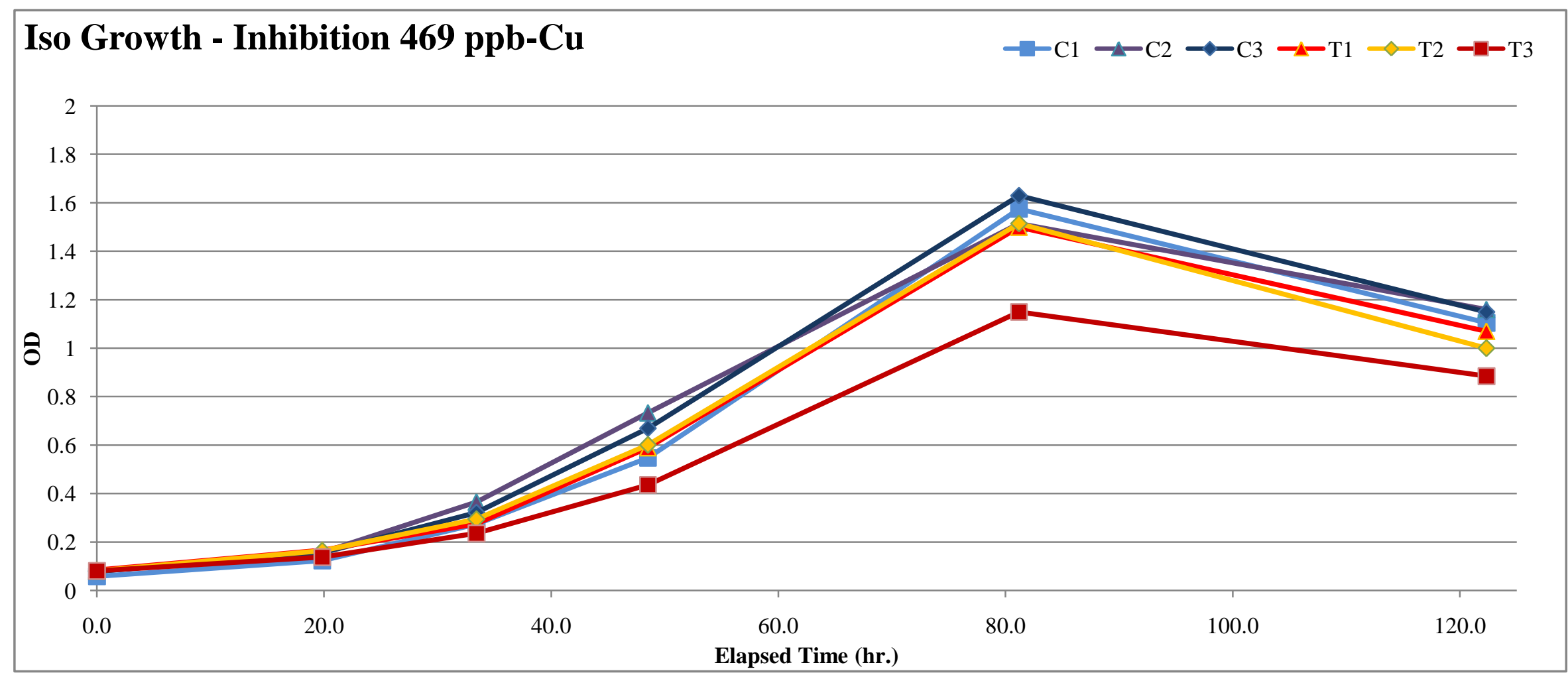

Figure 18: Induced inhibition test using $469 \mathrm{ppb}-\mathrm{Cu}$ on an algal strain isolated on BBM media with 1.7\% agar from the California Polytechnic State University's wastewater treatment ponds. C1-3 refer to the control cultures while T1-3 refer to the test cultures. 
The second induced inhibition test was conducted on January 28, 2014. Because no statistically significant inhibition was observed in the first experiment, the copper concentration exposed to the algae test cultures was increased to $1 \mathrm{mg} / \mathrm{L}$ as copper. Ward's Chlorella sp. was selected for this experiment due to its use as the primary strain for the regrowth experiments. $\mathrm{Cu} 1-3$ were the controls for this experiment while $\mathrm{Cu} 4-7$ contained an additional $1 \mathrm{ppm}-\mathrm{Cu}$. Due to the large variance between replicates, this experiment was disregarded and terminated early in the growth phase. The observed variance was most likely caused by the age of the inoculation culture. The inoculum was in the light limited region (refer to section 3.8.3 for more information regarding light limited growth) before being transferred. This has been observed in previous experiments to increase variance. Further, the initial growth measured in all of the test cultures with $1 \mathrm{ppm}-\mathrm{Cu}$ did not appear hindered, with exponential growth rates ranging from 0.588 to 1.039 day $^{-1}$. The range of exponential growth rates of the controls was 0.754 to 0.978 day $^{-1}$. Therefore, it was determined the copper concentration would need to be further increased to observe significant inhibition.

To obtain an idea of the copper concentration required to significantly impair Ward's Chlorella sp., the final induced inhibition experiment employed a higher range of copper concentrations. Three controls and four experimental cultures were inoculated on February 12, 2014 (Figure 19). The test cultures contained copper concentrations of 5, 10, 50, and $100 \mathrm{ppm}-\mathrm{Cu}$ (Figure 20).

An analysis of the collected data from the experiment found a decreasing normalized growth rate between 5 and $10 \mathrm{ppm}-\mathrm{Cu}$ (Figure 21). This pattern could signify that, at copper concentrations in the range of $10 \mathrm{ppm}$, induced toxicity occurs within the culture. 
As the copper concentration was further increased to 50 and $100 \mathrm{ppm}$, the data suggest an increase in the normalized growth rate and the 100 ppm culture appeared to grow approximately $45 \%$ better than the controls, based on OD. However, visual and microscopic observation showed that the 50 and 100 ppm cultures were extremely flocculated and tinted blue. This blue color came from the additional copper introduced to the culture. The initial spike of copper effectively killed the algae cells in the experimental cultures due to the high toxic effect of copper. The subsequent optical density measurements apparently rose due to increasing degradation of the algal cells which produced light absorbing cell fragments. It was these small fragments which increased the optical density of the two cultures with high copper concentrations and resulted in measurement of a false increased growth rate. 


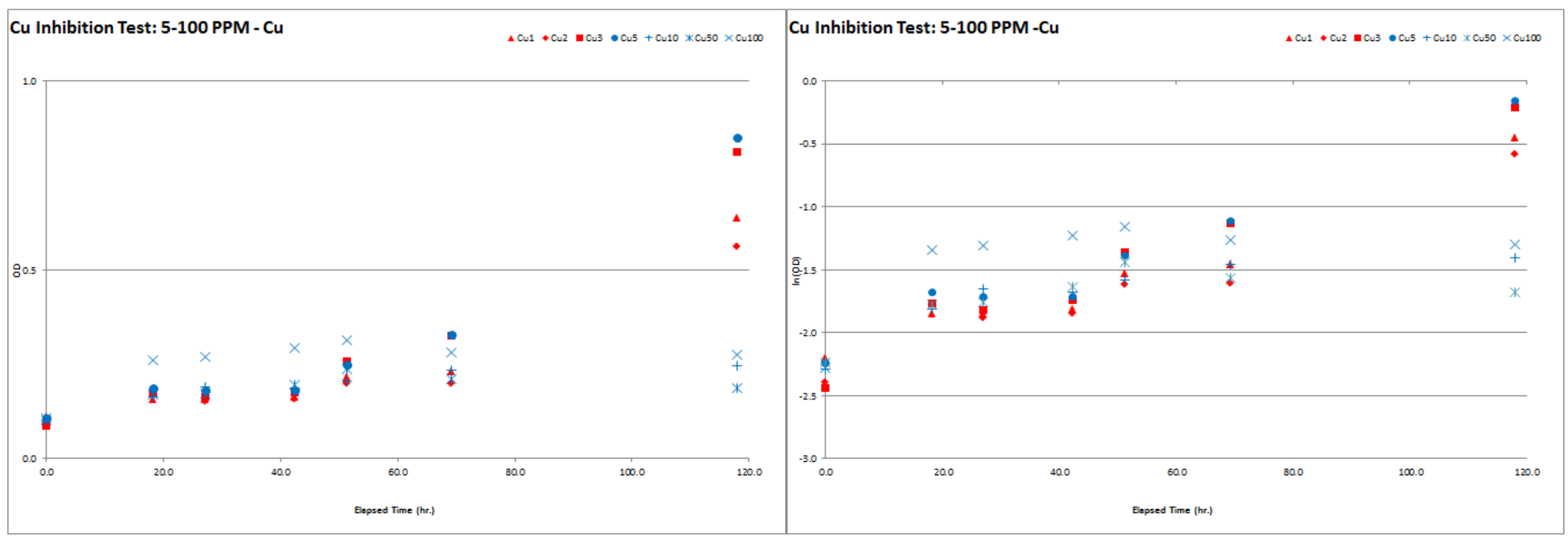

Figure 19 (Top): The growth curves for the third induced inhibition experiment. The controls are red while the experimental cultures are blue.

Figure 20 (Bottom, center): Level of

$\mathrm{Cu}$ addition and the observed

maximum specific growth rate (per day). The highlighted growth rate was determined to be an outlier and omitted from the analysis of the experiment (resides farther than 1.5 times the interquartile range).

Figure 21 (Bottom, right): The exponential growth rates for each test culture were normalized to the average exponential growth rate of the control cultures. The resulting graphic shows a decreasing normalized growth rate from 5 to $10 \mathrm{ppm}-\mathrm{Cu}$.

\section{Comparison of Cu Induced Toxicity}

\begin{tabular}{|c|c|c|}
\hline Culture ID & $\begin{array}{c}\text { Additional Copper } \\
\text { Added (PPM) }\end{array}$ & \begin{tabular}{|c} 
Exp Growth \\
Rate
\end{tabular} \\
\hline Cu1 & \multirow{3}{*}{$\begin{array}{l}\text { None. Control } \\
\text { Cultures }\end{array}$} & 0.4644 \\
\hline Cu2 & & 0.8136 \\
\hline Cu3 & & 0.8880 \\
\hline Cu5 & 5 & 0.7392 \\
\hline Cu10 & 10 & 0.5736 \\
\hline Cu50 & 50 & 0.6000 \\
\hline Cu100 & 100 & 1.2312 \\
\hline
\end{tabular}

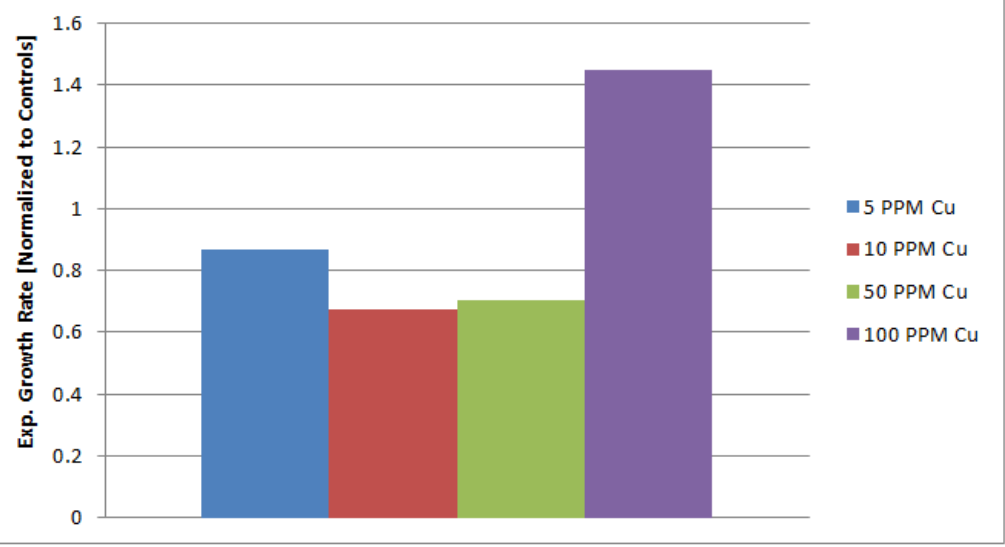

*High growth rates recorded for 50 and 100 PPM Cu were attributed to inaccurate OD measurements caused by enhanced floculation within the cultures. 


\subsection{Regrowth Experiments}

Several individual regrowth experiments were conducted over the course of the research. Through these trials, modifications were made to the regrowth methodology to allow for collection of meaningful data. Ultimately, a finalized methodology was created and carried out on ten cultures of Ward's Chlorella sp. Further, an experiment looking into the growth potential of anaerobically digested lysed algal biomass was conducted with the intention of supplementing large-scale algae ponds with this nutrient slurry to demonstrate nutrient recycling for algae growth.

\subsubsection{Regrowth on Bold's Basal Medium (3N3P) with Vitamins and Bicarbonate}

As discussed previously in the media optimization section of the results, it was discovered that restoration of all trace metals and micronutrients did not impair growth. After a preliminary regrowth trial in which the micronutrients were restored to several of the cultures, it was also determined that the restoration of these nutrients was required between rounds of growth. Although there was no substantial effect on the maximum specific growth rates, there was a notable difference in the color of the cultures. A photograph was taken at the end of this preliminary trial (Figure 22). The cultures with trace metals and micronutrients restored grew to a much richer green color, which was similar to the color of the controls. After this observation, the trace metal spike experiment was conducted which ascertained there was no effect caused from the introduction of $8 \mathrm{x}$ normal trace metal/micronutrients into a culture (refer to section 4.2.3 Trace Metal and Micronutrient Spike). 


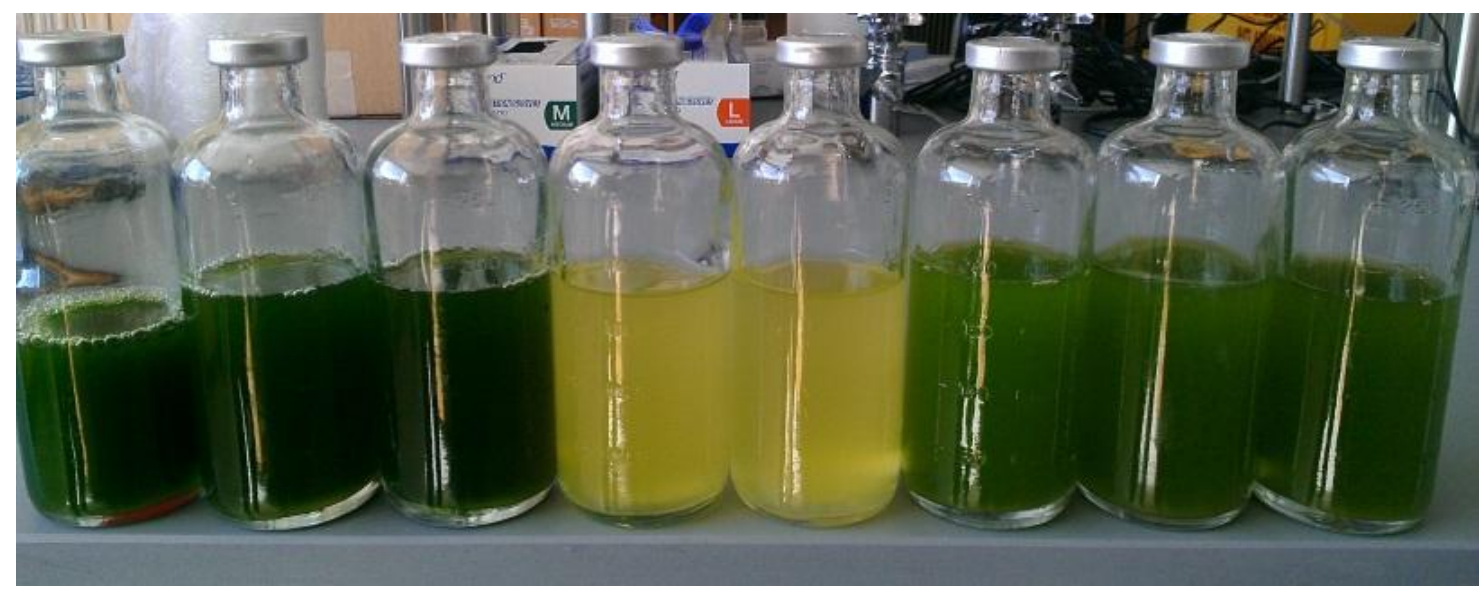

Figure 22: Round 3 of growth during a preliminary regrowth experiment. From left to right: $x 3$ controls, $\mathrm{x} 2$ experimental cultures with only nitrate and phosphate restored, $\mathrm{x} 3$ experimental cultures with nitrate, phosphate, and trace nutrients restored.

On March 31, 2014, the first round of ten experimental bottles was inoculated. They were allowed to grow to stationary phase, which occurred on April 6, 2014 (Figure 23). The average, maximum specific growth rates for A1-5 and A6-10 were 0.702+/-0.07913 and $0.6936+/-0.05725$ day $^{-1}$, respectively. The specific growth rates of the replicates were grouped well throughout the experiment, with a relative standard deviation of $11.3 \%$ and $8.3 \%$ for A1-5 and A6-10, respectively. 


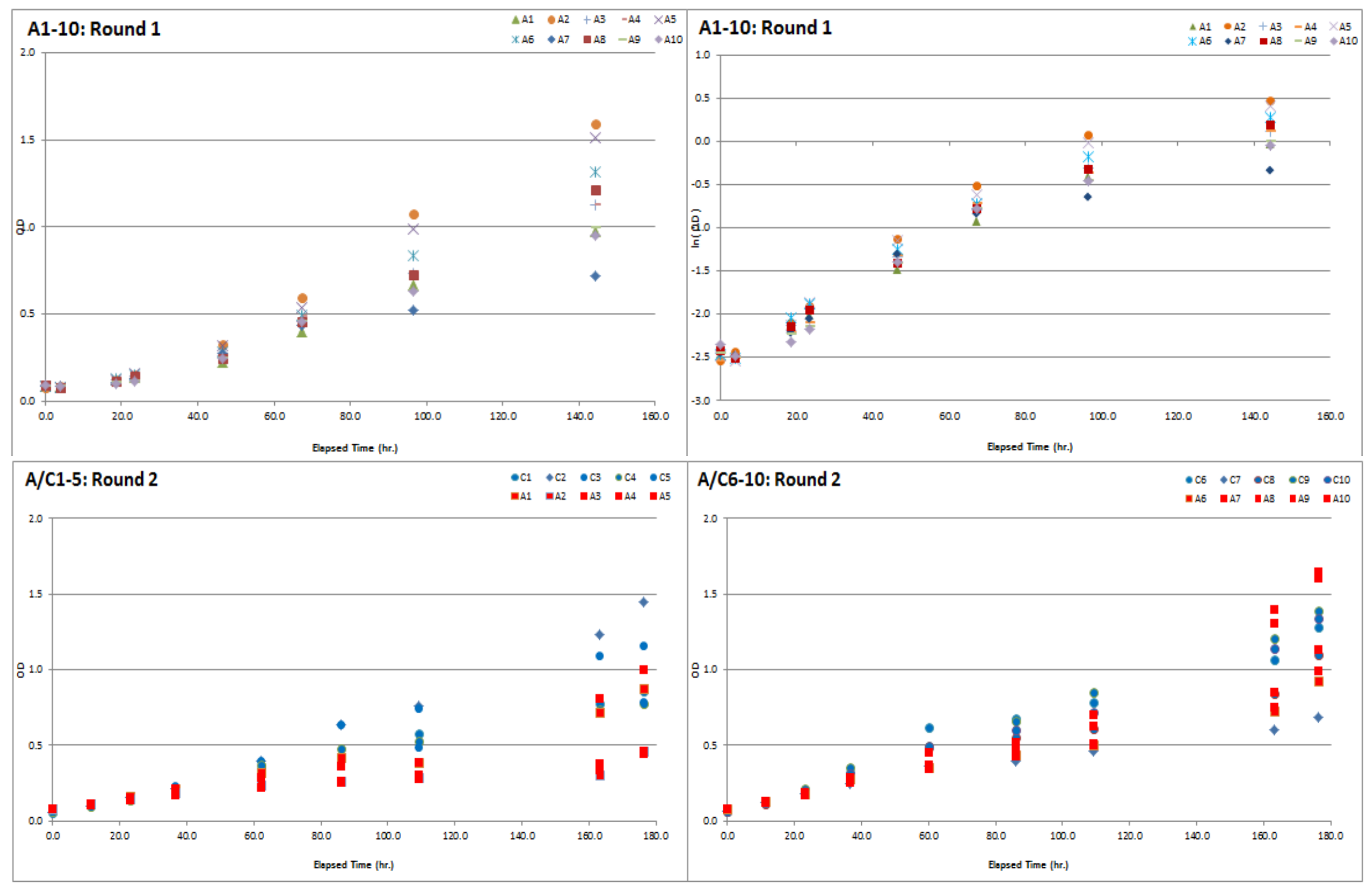

Figure 23 (Top): Round 1 growth of experimental cultures A1-10. Cultures were inoculated on March 31 and harvested on April 6, 2014.

Figure 24 (Bottom): Round 2 growth data of cultures A1-10. They were separated into A1-5 and A6-10 due to the cultures residing on different stir plates. Each group of five experimental bottles and had an equal number of controls on the respective stir plate. 
Another potential concern was the intense color of the media after the cells were harvested (Figure 25). Samples of the harvested and filtered "used" were collected and compared to D.I. water and Bold's Basal Medium using a spectrophotometer at $750 \mathrm{~nm}$. It was determined that an insignificant difference in the OD, less than $3 \%$, existed between each of these solutions. Therefore, optical density could still be used as the primary measurement device of biomass for the regrowth experiments.

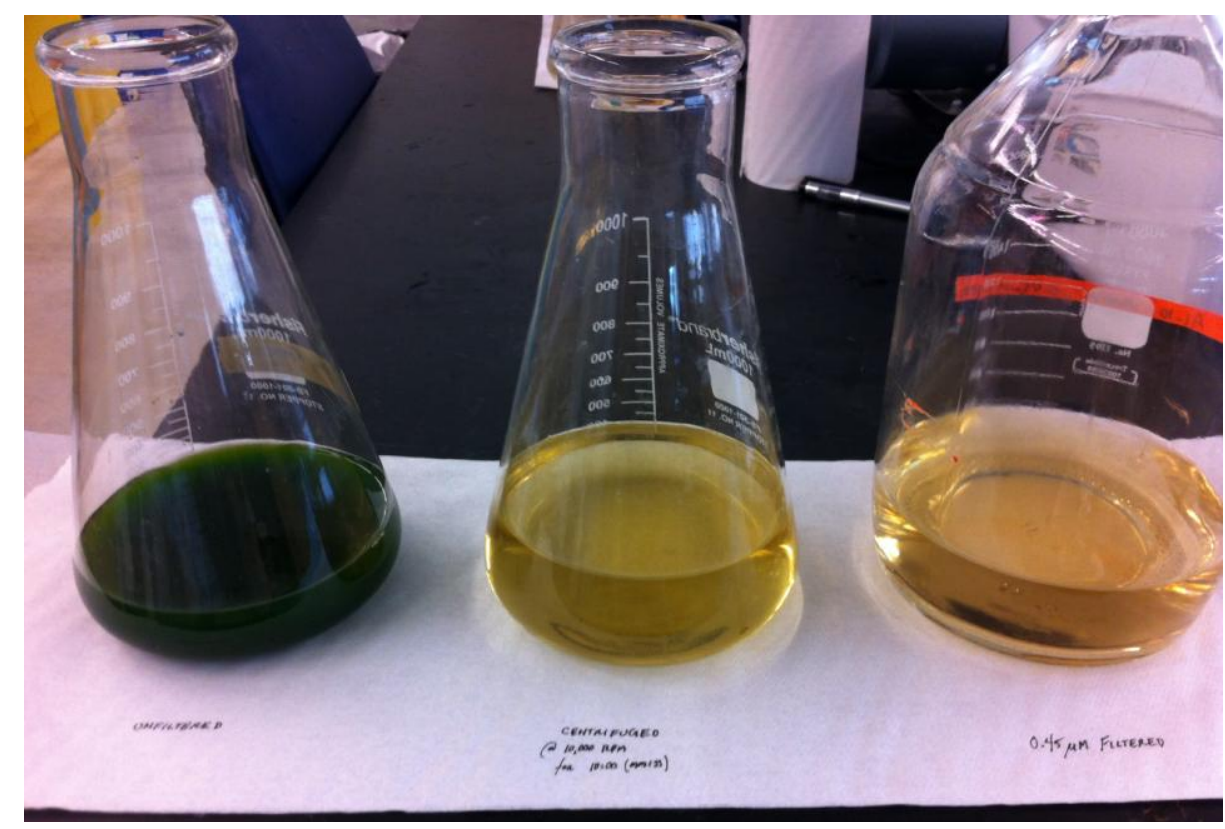

Figure 25: Various stages of harvesting of Round 1 algae cultures grown in BBM (3N3P + Vitamins + Bicarbonate) (A1-10). From left to right: unfiltered, centrifuge supernatant, 0.45 micrometer filtered.

Both nitrate and dissolved reactive phosphorus were measured using the procedures outlined in Section 3.6 Nutrient Analysis. Nitrate was determined to be 95.3\% lacking while phosphorus was $21.4 \%$ depleted when compared to the original concentrations in BBM (3N3P + Vitamins + Bicarbarbonate). These nutrients were restored to initial Bold's Basal Medium (3N3P) concentrations. Trace metals, micronutrients, and vitamins were added back in full concentration. The restored media was then passed through a 
0.22 micrometer pore size syringe filter into a sterile reagent bottle and stored at $10^{\circ} \mathrm{C}$ in the dark.

Once the stock algal cultures were ready, the second round of growth was inoculated on May 5, 2014. Because this was the second round of growth, an equal number of controls were grown alongside the experimental cultures. Maximum specific growth rates were normalized using the respective average growth rates of the controls to allow a comparison to be made between Round 1 and Round 2 growth rates. Round 2 cultures required a longer growth period to reach stationary phase (Figure 24). Replicate maximum specific growth rates also grouped tightly for this round of growth with a relative standard deviation of approximately $8.4 \%$ for both sets of cultures. Figure 26 shows a comparison of growth rates for Round 1 and Round 2. The decrease in specific growth rate between the Round 2 cultures and the controls was determined to be significant through a student's t-test with both p-values less than 0.001 . Further, a significant difference also existed between the normalized growth rates for the Round 1 and Round 2 cultures with p-values less than 0.001 for both sets of cultures. 


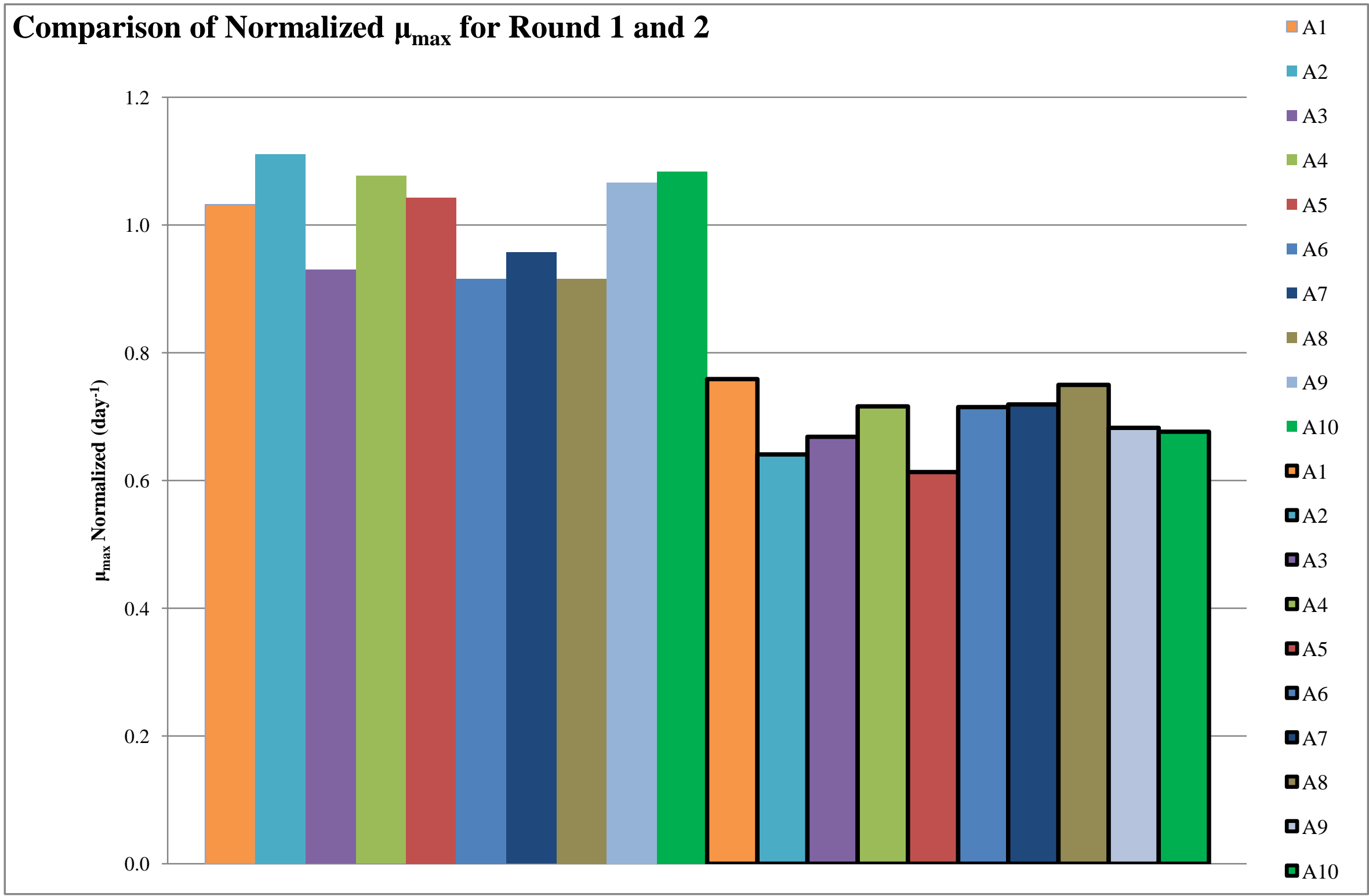

Figure 26: Growth rates determined for the experimental cultures for both round 1 and 2 of growth. The growth rates were normalized to the average growth rate for controls to allow for unbiased comparison. Round 2 growth rates were outlined with a black border for distinction. 


\subsubsection{Regrowth on Anaerobically Digested Lysed Algal Biomass}

Anaerobic digester effluent was collected from Alex Hill, a fellow researcher at California Polytechnic State University (Hill, 2014). The filtrate of anaerobicallydigested, homogenized algal biomass (digestate) had a similar color as the filtered "used" Bold's Basal Media from regrowth experiments. An image of the filtration steps of the digestate was also included in section 4.5 (Figure 29).

The digestate was diluted to $50 \%$ (v/v) using a saline make up of sodium chloride, magnesium sulfate, and copper chloride. This was done to mimic the main salts present in Bold's Basal Medium. On April 1, 2014, five digestate columns were inoculated using Ward's Chlorella sp. which was previously acclimated to ammonia as a nitrogen source. A photograph of the columns immediately after inoculation was included as Figure 27.

The columns were allowed to grow for nine days. During this time the controls and digestate columns achieved an average maximum specific growth rate of 1.79 and 2.41 doublings per day, respectively. There was a low relative standard deviation between all replicates at approximately $3 \%$ signifying a high degree of grouping. Interestingly, the digestate columns had a significantly higher maximum specific growth rate, which was confirmed by a student's t-test with a resultant $\mathrm{p}$-value of $1.1 \times 10^{-7}$. Figure 28 shows a photograph of the columns taken on April 3, 2014. A notable color difference exists between the controls and the digestate columns. Initially this was thought to be a sign of a nutrient limitation within the digestate cultures 


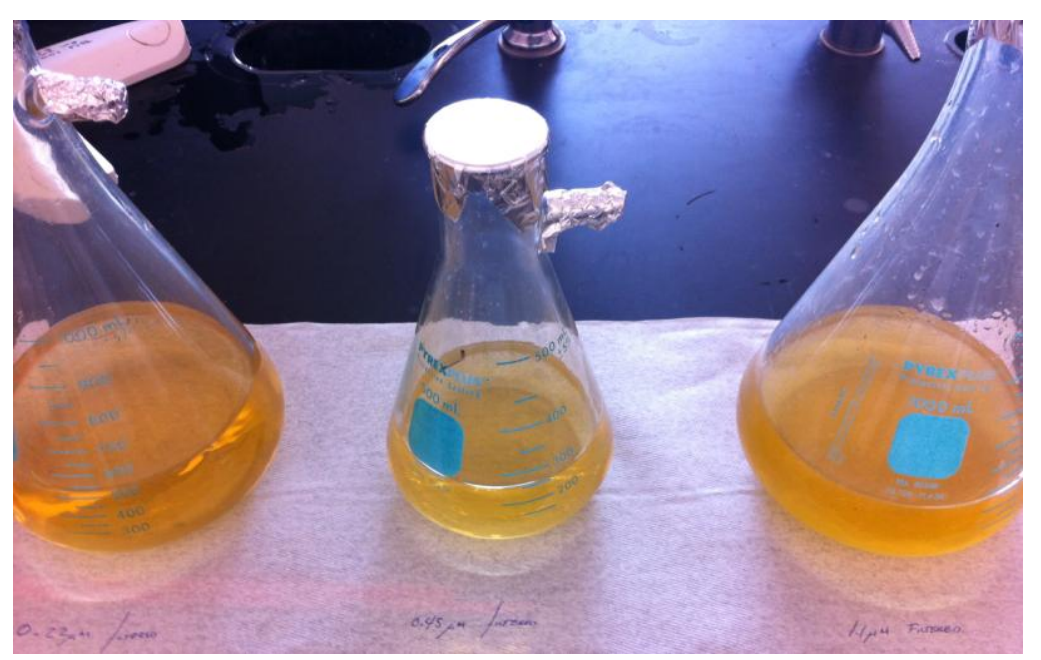

Figure 27 (Above): Various stages of anaerobically digested algal biomass. From left to right: 0.22 micrometer filtered, 0.45 micrometer filtered, 1.1 micrometer filtered.
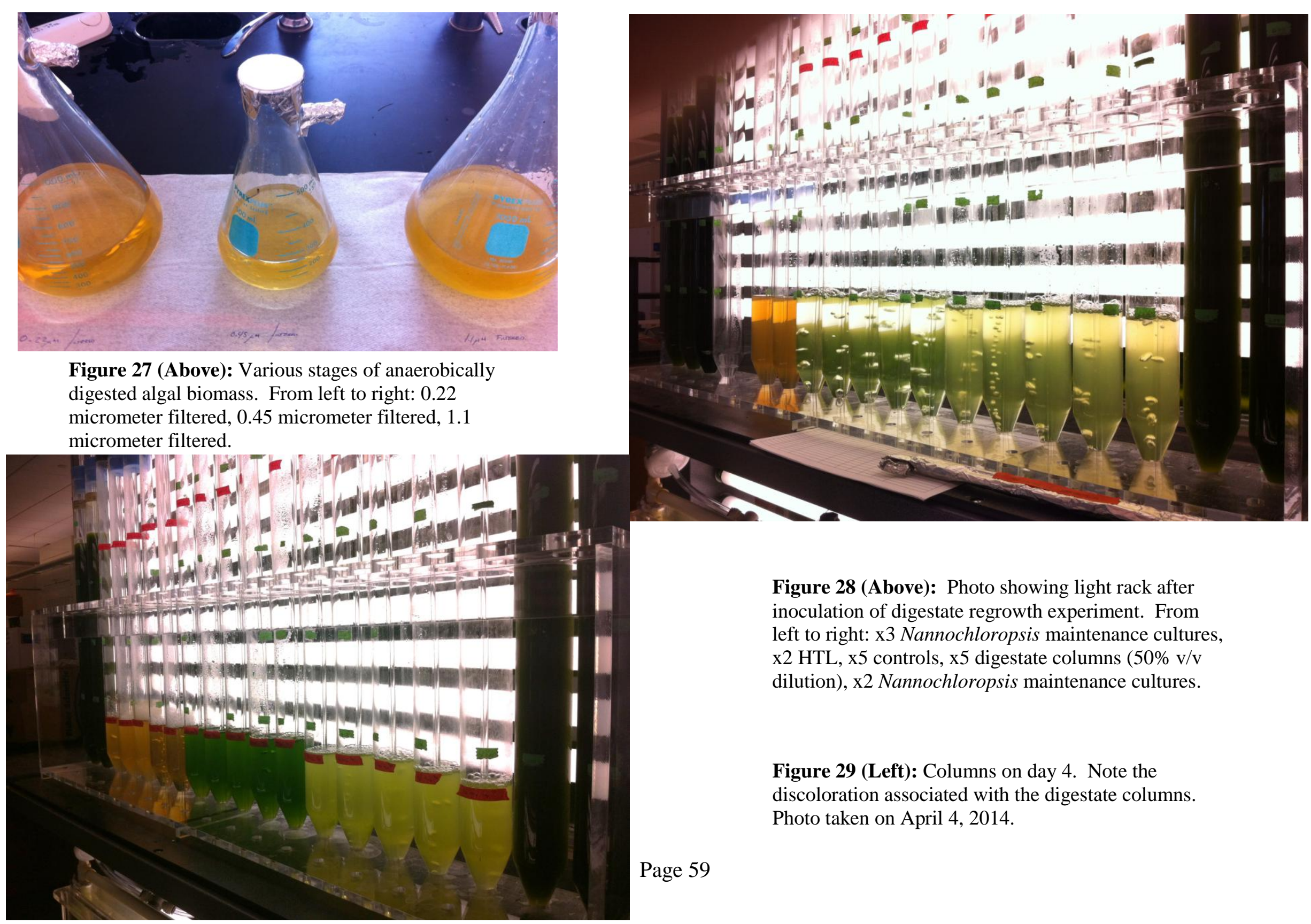

Figure 28 (Above): Photo showing light rack after inoculation of digestate regrowth experiment. From left to right: $\mathrm{x} 3$ Nannochloropsis maintenance cultures, $\mathrm{x} 2$ HTL, $\mathrm{x} 5$ controls, $\mathrm{x} 5$ digestate columns $(50 \% \mathrm{v} / \mathrm{v}$ dilution), $\mathrm{x} 2$ Nannochloropsis maintenance cultures.

Figure 29 (Left): Columns on day 4. Note the discoloration associated with the digestate columns. Photo taken on April 4, 2014.

Page 59 
On April 7, 2014, all trace metals and micronutrients were added to the digestate columns. Typically $8 \mathrm{~mL}$ of trace metals and micronutrients are added per liter of Bold's Basal Medium. Therefore, to keep the proportions equal to that of the control columns, $1.6 \mathrm{~mL}$ was added to each $200 \mathrm{~mL}$ digestate column. The digestate columns were allowed to grow an additional 3 days due to increased growth (Figure 30).

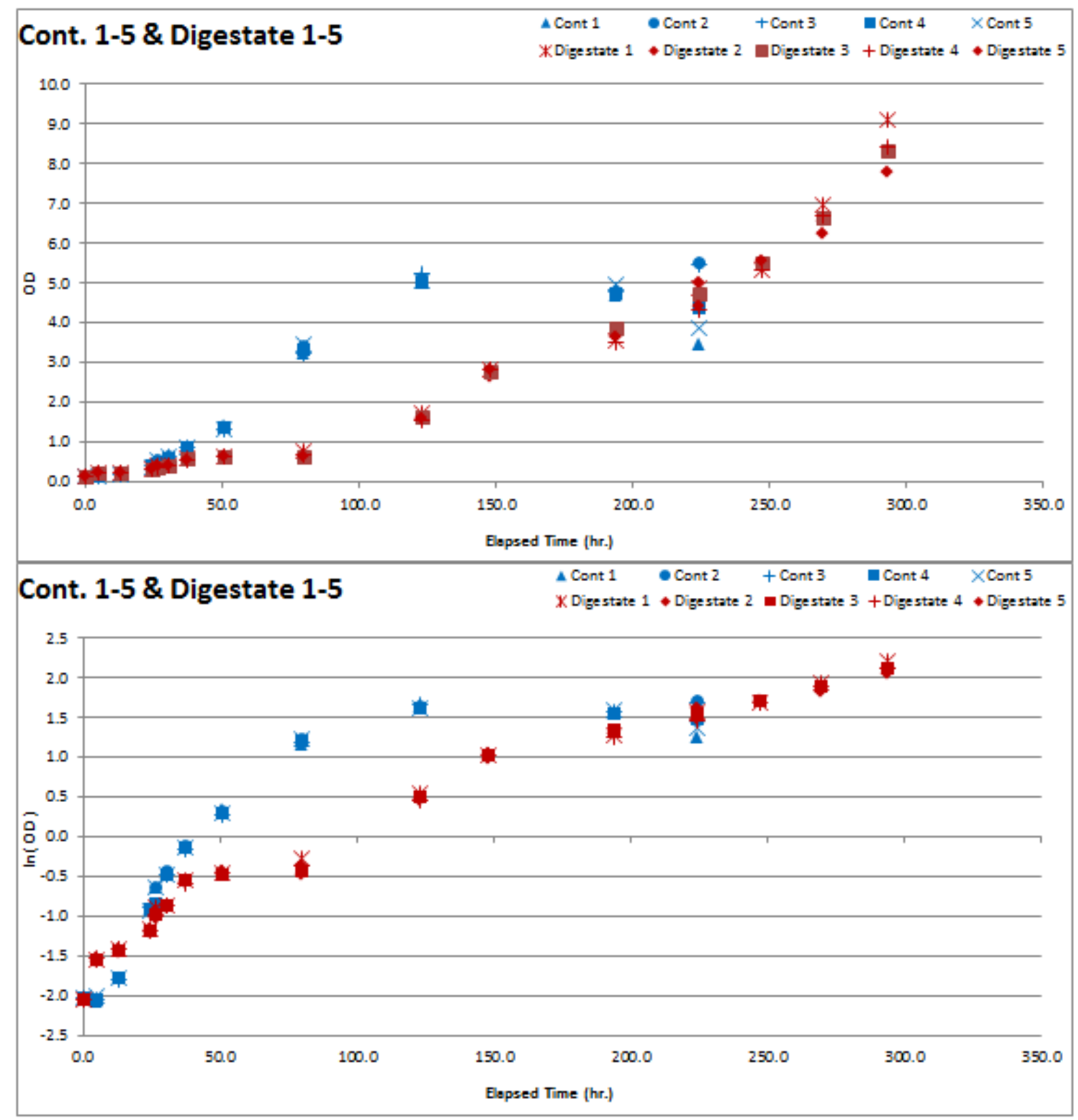

Figure 30: Growth data collected during the digestate growth potential experiment. Blue denotes control cultures while red denotes experimental cultures with $50 \%(\mathrm{v} / \mathrm{v})$ digestate. 
The control columns (blue) show a classic microbial growth curve with notable exponential, light limited and stationary phases. The digestate columns (red), however, do not follow a typical growth regime. There was an initial exponential jump in density of the columns that was complete by 50 hours. At a time of 100 hours, the cultures again began growing but at a decreased rate compared to the initial exponential jump. After the addition of the trace metal and micronutrients at a time equal to 150 hours, the cultures continued to grow until the experiment was ended at 300 hours. This was due to exhaustion of all the culture volume through the sampling events.

Although the addition of the micronutrients and trace metals did not produce an exponential jump in the growth of the digestate columns, there was a significant change in the color of the cultures. After the addition, the cultures gained the forest green color typically associated with green algae. Figure 31 shows a photograph taken on April 17 of

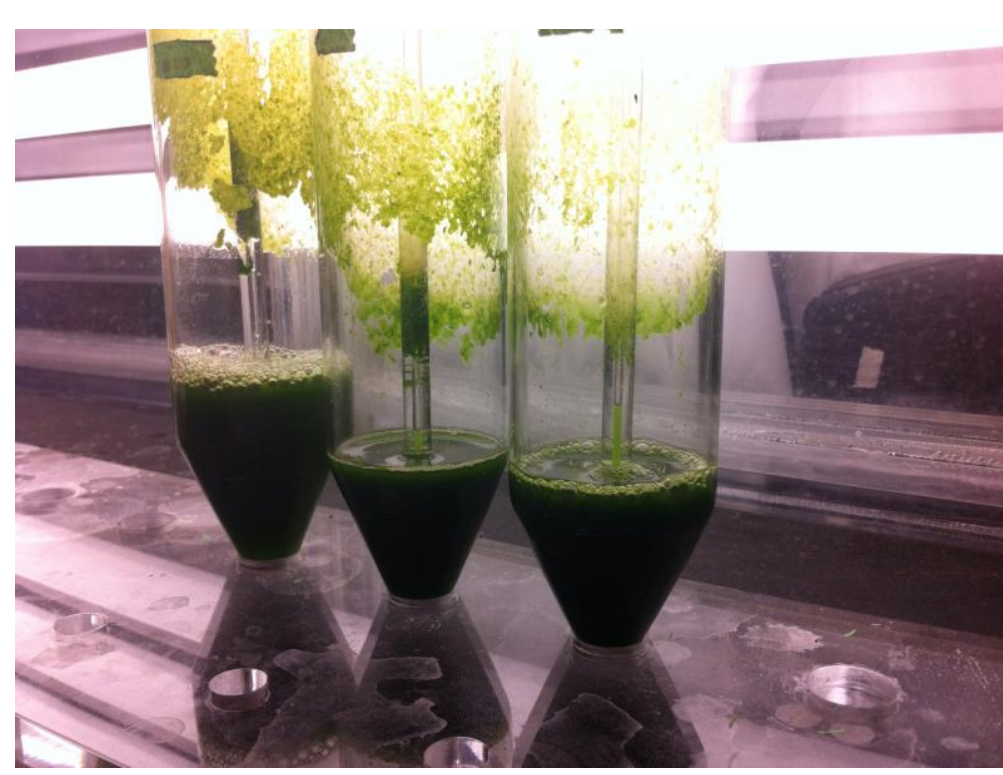

Figure 317: Photograph of the digestate columns on April 17, 2014. The columns gained the dark, green color after the addition of trace metals and micronutrients. several of the digestate columns. 


\subsection{CONCLUSIONS}

The following section discusses derived conclusions from the experiments conducted throughout the research and outlines recommended future studies.

\subsection{Bold's Basal Medium (3N3P with Vitamins and Bicarbonate) Regrowth Experiment}

Data collected during the second round of growth on Bold's Basal Medium confirmed there was a significant difference in the maximum specific growth rates between rounds of growth. Further, there was also a significant difference between the growth rates of the second round of experimental and control cultures which further demonstrates the presence of inhibition. A third round of growth should be conducted on this media to determine if the degree of inhibition will increase further.

The reintroduction of micronutrients, trace metals, and vitamins into the "used" culture media prior to inoculating the second round of growth was critical. Through this addition, there was no doubt that the cultures were not limited on any of the nutrients present in Bold's Basal Medium (3N3P). Therefore, the noted inhibition must be due to the presence of a toxin or allelochemicals produced by Ward's Chlorella sp.

\subsection{Anaerobically Digested Algal Biomass Growth Potential Experiment}

The conducted digestate growth experiment provided good insight regarding possible future applications. From the collected data it can be concluded that anaerobically digested lysed algal biomass can function as the primary source of nutrients for algal growth. Further, the digestate actually showed an increased initial growth rate compared to the Bold's Basal Medium controls (with ammonia in place of nitrate) as well as 
increased growth duration. It is possible the increased duration of growth was due to the introduction of micronutrients and trace metals at a culture time equal to 150 hours. Regardless, the digestate columns were lacking one or more of those nutrients which caused the algae to continue to grow much longer than the BBM controls.

\subsection{Recommended Future Studies}

The main focus of this research was the optimization of growth parameters in order to allow for valid regrowth potential experiments to be conducted. Therefore, it is recommended that these experiments be conducted in accordance with the parameters outlined by this research. Incorporation of higher intensity lights could decrease growth variance between replicates by achieving saturating lighting conditions within the culture vials.

The growth potential experiment conducted on the digester effluent was a preliminary experiment that needs to be repeated. It is recommended that this experiment be replicated but with micronutrients and trace metals added into the digestate cultures from the beginning. It was confirmed through the initial study that these nutrients allowed for increased growth within the digestate columns. Further, the preliminary experiment was conducted using $50 \%(\mathrm{v} / \mathrm{v})$ of digestate that was diluted with a saline makeup. By using full strength digestate in the next experiment, the future use of digestate as a nutrient substitute for defined media can be determined. It is possible that use of the full strength digestate may require a new biomass measurement such as volatile suspended solids (VSS) or chemical oxygen demand (COD) due to the inherent color of the digestate. This would also require an increase in culture volume to allow for the required additional sampling volume for VSS. 


\section{REFERENCES}

American Public Health Association. (2006). Standard Methods for the Examination of Water and Wastewater. American Water Works Association, and Wate Environment Federation.

Bacterial Growth and Regulation. (2007, Jan 28). Retrieved Jun 1, 2014, from Crondon: http://cronodon.com/BioTech/Bacteria_Growth.html

Bischoff, H. B. (1963). Some Soil Algae From Enchanted Rock and Related Algal Species. Physcological Studies IV, 1-95.

Dauta A., D. J. (1990). Growth rate of four freshwater algae in relation to light and temperature. Hydrobiologia, 207, 221-226.

Doan N.T., R. R. (2000). Allelopathic actions of the alkaloid 12-epi-hapalindole E isonitrile and calothrixin A from cyanobacteria of the genera Fischerella and Calothrix. Journal of Applied Phycology, 12, 409-416.

Franklin N.M., S. J. (2002). Effect of initial cell density on the bioavailability and toxicity of copper in microalgal bioassays. Eviron Toxicol Chem, 4, 742-751.

Freshwater Alga and Cyanobacteria, Growth Inhibition Test. (2006, March 23). OECD GUIDELINES FOR THE TESTING OF CHEMICALS.

Gleason, F. B. (1986). Activity of the natural algicide, cyanobacterin, on eukaryotic microorganisms. FEMS Microbiology Letters, 33, 85-88.

Goldman J., O. W. (1974). The kinteicks of inorganic carbon limited algal growth. Journal Water Pollution Control Federation, 554-574.

Graneli, E. H. (2006). Allelopathy in Harmful Algae: A Mechanism to Compete for Resources? Ecological Studies, Vol. 189, 189-201.

Henze, M. C. (2008). Biological Wastewater Treatment: Principles, Modeling and Design. London, U.K.: IWA Publishing.

Ikawa, M. S. (1997). Inhibition of Chlorella growth by degradation and related products of linoleic and linolenic acids and the possible significance of polyunsaturated fatty acids in phytoplankton ecology. Hydrobiologia, 356, 1430148.

Juttner F, W. J.-T. (2000). Evidence of allelochemical activity in subtropical cyanobacterial biofilms of Taiwan. Archiv fur Hydrobiologie, 147, 505-517.

Kearns K.D., H. M. (2000). Green algal extracellular products regulate antialgal toxin production in a cyanobacterium. Environmental Microbiology, 2, 291-297.

Kohn RA, D. T. (1998). Calculation of the Buffering Capacity of Bicarbonate in the Rumen and In Vitro. Journal of Animal Sciences, 1702-09. 
Leflaive, J. T.-H. (2007). Algal and cyanobacterial secondary metabolites in freshwaters: a comparison of allelopathic compounds and toxins. Freshwater Biology, 52, 199214.

Legrand, C. R. (2003). Allelopathy in phytoplankton - biochemical, ecological, and evolutionary aspects. Phycologia, 42, 406-419.

Mandalam R., P. B. (1998). Elemental Balancing of Biomass and Medium Composition Enhances Growth Capacity in High-Density Chlorella vulgaris Cultures. BIOTECHNOLOGY AND BIOENGINEERING, 59, 605-611.

McCracken M., M. R. (1979). A Chemical Characterization of an Algal Inhibitor Obtained from Chlamydomonas. Department of Biology, Texas Christian university, Fort Worth, Texas, 272-276.

Moody J., M. C. (2014). Global evolution of biofuel potential for microalgae. Preceedings of the National Academy of Sciences.

Price N., H. G. (1988). Preparation and Chemistry of the Artificial Algal Culture Medium Aquil. Biological Oceanography, 6, 443-461.

Rachlin J., G. A. (1991). The Effects of pH on the Growth of Chlorella vulgaris and Its Interactions with Cadmium Toxicity. Archives of Environmental Contamination and Toxicology, 20, 505-508.

Radich, A. (2004, Jun 08). Biodiesel Performance, Cost, and Use. Retrieved May 05, 2014, from Energy Information Administration: http://www.eia.gov/oiaf/analysispaper/biodiesel/

Sasi D., H. G. (2009). Effect of LIght Intensity on Growth of Chlorella vulgaris in a Novel Circulating Loop Photobioreactor. Department of Chemical Engineering, Univirsity of Saskatchewan, Saskatoon, Saskatchewan, Canada.

Uggetti E., S. B. (2013). Anaerobic digestate as a substrate for microalgae culture: The role of ammonium concentration on the microalgae productivity. Bioresource Technology, 152, 437-443.

Vardi A., B.-F. I. (1999). Programmed cell death of the dinoflagellate Peridinium gatunense is mediated by Co2 limitation and oxidative stress. Current Biology, 9 , 1061-1064.

von Elert E., J. F. (1996). Factors influencing the allelopathic activity of the planktonic cyanobacterium Trichormus doliolum. Phycologia, 35, 68-73.

Zheng-Rong O., X.-B. W.-H.-J.-Y.-G. (2010). The Effects of Light Intensities, Temperatures, $\mathrm{pH}$ and Salinities on Photosynthesis of Chlorella. Journal of Wuhan Botanical Research, 28, 49-55. 


\section{Appendix A $\quad$ : Summary of Spreadsheet Titles of Experimental Data}

All data located in the "CHENVE" google drive, and in the "Exp Inhibition" subfolder.

Media Optimization:

Carrying Capacity Spreadsheet: "WW Media Biomass Carrying Capacity\&Nutrient Additions_CB_5_12_cb"

Vitamin Additions: "2_4_14_BBMvsMODBBM_Iso8_CB"

$\underline{\text { Trace Metal and Micronutrient Spike }}$

x 8 Spike: "3_25_14_Ward22-23_TraceSpike8x"

Induced Inhibition:

469ppb - $\mathrm{Cu}$ (ISO): "10_28_13_Iso Inhibition $\mathrm{CuCl}_{2}$ "

$1 \mathrm{ppm}-\mathrm{Cu}$ (Ward's): "1_28_14_Cu_Inhibition_Test_1PPM"

5-100ppm-Cu (Wards's): “2_12_14_CuTest_CB"

Regrowth Experiments:

BBM3N3P + Vits. + Bicarb:

Round 1: "3_31_14_A1-10_Ward24,25"

Round 2: "5_14_14_A1-5_round2" \& "5_14_14_A6-10_round2"

Digestate Potential: "4_1_14_Digestate_cb" 